Claremont Colleges

Scholarship@Claremont

WM Keck Science Faculty Papers

W.M. Keck Science Department

4-1-1996

\title{
Oscillatory Bifurcation with Broken Translation Symmetry
}

Adam S. Landsberg

Claremont McKenna College; Pitzer College; Scripps College

Edgar Knobloch

University of California - Berkeley

\section{Recommended Citation}

Landsberg, A.S., and E. Knobloch. "Oscillatory Bifurcation with Broken Translation Symmetry." Physical Review E 53.4 (1996): 3579-3600. DOI: 10.1103/PhysRevE.53.3579

This Article is brought to you for free and open access by the W.M. Keck Science Department at Scholarship @ Claremont. It has been accepted for inclusion in WM Keck Science Faculty Papers by an authorized administrator of Scholarship @ Claremont. For more information, please contact scholarship@cuc.claremont.edu. 


\title{
Oscillatory bifurcation with broken translation symmetry
}

\author{
A.S. Landsberg* and E. Knobloch \\ Department of Physics, University of California, \\ Berkeley, California 94720
}

(Received 27 December 1994; revised manuscript received 26 June 1995)

\begin{abstract}
The effect of distant endwalls on the bifurcation to traveling waves is considered. Previous approaches have treated the problem by assuming that it is a weak perturbation of the translation invariant problem. When the problem is formulated instead in a finite box of length $L$ and the limit $L \rightarrow \infty$ is taken, one obtains amplitude equations that differ from the usual Ginzburg-Landau description by the presence of an additional nonlinear term. This formulation leads to a description in terms of the amplitudes of the primary box modes, which are odd and even parity standing waves. For large $L$, the equations that result take the form of a Hopf bifurcation with approximate $D_{4}$ symmetry. These equations are able to describe, qualitatively, not only traveling and "blinking" states, but also asymmetrical blinking states and "repeated transients," all of which have been observed in binary fluid convection experiments.
\end{abstract}

PACS number(s): 47.20.Bp, 47.20.Ky, 03.40.Kf

\section{INTRODUCTION}

This paper is devoted to the understanding of the effects of breaking translation invariance in continuum systems undergoing a bifurcation to traveling waves. We imagine that the translation invariance is broken by the presence of endwalls of some container but assume that these are distant so that their effects might be expected, in some appropriate sense, to be weak. We focus on the effects of such endwalls near the onset of the instability. The two canonical examples of systems of this type are provided by doubly diffusive (or binary fluid) convection and by spiral vortices in the Taylor-Couette system with counter-rotating cylinders. Since a pure traveling wave cannot exist in a finite container it is clear that the endwalls must be responsible for substantial modification of the initial instability. Experimentally one finds the following (e.g., [1-4]):

1. Traveling waves. Surprisingly, propagating waveforms analogous to the pure traveling wave patterns found in unbounded systems can emerge in finite systems with endwalls. Within a limited portion of the container the appearance of these waves can resemble that of pure traveling waves. Both left and right propagating patterns are possible, depending on initial conditions. Such waves, in the form of spiral vortices, are well known in the Taylor-Couette system, and have been extensively studied in the context of binary fluid mixtures [1-3].

2. Blinking states. The presence of endwalls can induce so-called "blinking states," in which the direction of propagation of the wave reverses. Such reversals can be either periodic or irregular, depending upon system parameters [2-4]. Such waves were first discovered in the

*Permanent address: School of Physics, Georgia Institute of Technology, Atlanta, GA 30332. form of "alternating" spiral vortex flow in the TaylorCouette system [5] and in numerical simulations of doubly diffusive convection [6].

3. Repeated transients/collapse states. In binary mixture experiments an additional phenomenon, dubbed "repeated transients," has also been observed [7]. In this state a small-amplitude traveling wave solution grows in amplitude (maintaining its spatial profile), and then undergoes a rapid collapse back to a small-amplitude state. This process repeats at irregular intervals. This phenomenon depends sensitively on the aspect ratio of the system. A similar phenomenon was observed by Jacqmin and Heminger in a numerical study of binary fluid convection in a rectangular container [8], with the primary mode (apparently an even parity standing wave) growing into a large-amplitude state (dominated by a small number of spatial modes), and then undergoing a sudden, rapid collapse. This process then repeats. These repeated transients and collapse states are not yet understood.

In an unbounded system with periodic boundary conditions the transition from the trivial state to oscillatory behavior is described by the Hopf bifurcation with $\mathrm{O}(2)$ symmetry. The normal form for this bifurcation, truncated at third order, is given by [9]

$$
\begin{aligned}
& \frac{d v}{d t}=(\lambda+i \omega) v+a|w|^{2} v+b\left(|v|^{2}+|w|^{2}\right) v, \\
& \frac{d w}{d t}=(\lambda-i \omega) w+\bar{a}|v|^{2} w+\bar{b}\left(|v|^{2}+|w|^{2}\right) w
\end{aligned}
$$

where $v, w$ denote the amplitudes of left- and righttraveling waves, and $a, b$ are complex coefficients. Solutions with $|v|=|w|$ are reflection symmetric and are hereafter called standing waves. This interpretation follows from the form of the temperature eigenfunction [10],

$$
\begin{aligned}
\vartheta(x, y, t)= & \mathbb{R}\left\{[v(t)+\omega(t)] e^{i k x} f(y)\right\} \\
& +(\text { higher-order terms) },
\end{aligned}
$$


where $f(y)$ describes its vertical structure. By construction these equations are equivariant under $(v, w) \rightarrow$ $e^{i k l}(v, w)$ (i.e., under translations $\left.x \rightarrow x+l\right)$, and $(v, w) \rightarrow(\bar{w}, \bar{v})$ (i.e., reflections $x \rightarrow-x)$. These operations generate the group $\mathrm{O}(2)$. An additional symmetry, called a normal form symmetry, is also present: $(v, w) \rightarrow\left(e^{i \phi} v, e^{-i \phi} w\right)$. Equations $(1 \mathrm{a}, \mathrm{b})$ have two types of nontrivial solutions, traveling and standing waves, at most one of which can be stable [9].

Equations $(1 \mathrm{a}, \mathrm{b})$ are strictly valid only when the wave number $k$ is fixed at its onset value as $\lambda$ increases, since, once the Rayleigh number is raised above its critical value, there is a whole band of wave numbers, all of which are unstable. This problem is typically resolved by allowing the traveling wave amplitudes in the stream function (2) to be modulated on a long spatial scale $X$ and a slow time scale $T\left[v=A(X, T) e^{i \omega t}, w=B(X, T) e^{-i \omega t}\right]$. An asymptotic analysis then leads to a set of coupled complex Ginzburg-Landau equations for $A$ and $B$, whose structure depends on the magnitude of the group velocity $[11,12]$.

As indicated by the experimental observations above, the corresponding situation for large but bounded systems is more complex than the unbounded case. In the last few years there have been several efforts to develop the theoretical underpinnings needed to describe the dynamics of large aspect ratio systems near the onset of an oscillatory instability.

Cross [13] has adopted an approach based on a multiple scale analysis. The result is a pair of coupled complex Ginzburg-Landau equations describing the slow evolution of the envelope functions for the traveling wave solutions,

$$
\begin{aligned}
& A_{T}=D A_{X X}+s A_{X}+\Lambda A+a|B|^{2} A \\
& +b\left(|A|^{2}+|B|^{2}\right) A, \\
& B_{T}=\bar{D} B_{X X}-s B_{X}+\bar{\Lambda} B+\bar{a}|A|^{2} B \\
& +\bar{b}\left(|A|^{2}+|B|^{2}\right) B,
\end{aligned}
$$

together with boundary conditions designed to take into account the effects of the distant endwalls:

$$
\begin{array}{r}
A-\epsilon\left(\mu_{1} A_{X}+\nu_{1} \bar{B}_{X}\right)=0, \quad B-\epsilon\left(\mu_{2} B_{X}+\nu_{2} \bar{A}_{X}\right)=0, \\
\text { at } X=L / 2, \\
A+\epsilon\left(\bar{\mu}_{2} A_{X}+\bar{\nu}_{2} \bar{B}_{X}\right)=0, \quad B+\epsilon\left(\bar{\mu}_{1} B_{X}+\bar{\nu}_{1} \bar{A}_{X}\right)=0, \\
\text { at } X=-L / 2 .
\end{array}
$$

Here $A, B$ are the complex amplitudes of the left- and right-traveling waves depending on the slow spatial and temporal scales $X, T ; D$ is a complex diffusion coefficient, $s$ a measure of the group velocity (assumed to be small), and $\Lambda$ a measure of the growth rate and frequency shift of the modes. The boundary conditions are taken to be linear and homogeneous, on the assumption that the mode amplitudes $A, B$ become small near the endwalls. The most general such boundary conditions depend on the complex reflection coefficients $\mu_{1,2}, \nu_{1,2}$, and follow from symmetry considerations. Higher order derivatives enter with higher powers of $\epsilon(\ll 1)$ and are neglected. See [11, 13-16] for details. Numerical simulations of these equations have revealed behavior that qualitatively resembles some of that found in the experiments, including traveling waves and blinking states.

A more direct approach to this problem was suggested Dangelmayr and Knobloch [16-18]. The basic idea is to model the effects of distant endwalls by introducing small, linear $\mathrm{SO}(2)$-breaking (i.e., translation-breaking) terms into the normal form equations describing the "perfect" problem [Eqs. (1a) and (1b)]. The most general equations of this type take the form

$$
\begin{aligned}
& \frac{d v}{d t}=(\lambda+i \omega) v+a|w|^{2} v+b\left(|v|^{2}+|w|^{2}\right) v+e \bar{w} \\
& \frac{d w}{d t}=(\lambda-i \omega) w+\bar{a}|v|^{2} w+\bar{b}\left(|v|^{2}+|w|^{2}\right) w+\bar{e} \bar{v}
\end{aligned}
$$

where, as before, the amplitudes $v, w$ are associated with left- and right-traveling wave disturbances in the system (analogous to the "pure" traveling wave solutions found in the unbounded case), and $e$ is a (small) complex coefficient.

This system has been analyzed in considerable detail [18]. Owing to the symmetry-breaking terms, pure traveling waves are no longer possible. Instead, the primary instability is to an even or an odd standing wave solution. Depending on the spatial eigenfunctions these standing waves can take the form of "chevrons," with left-traveling waves dominating in the left half of the container and right-traveling waves dominating in the right half [16]. See [8] for explicit calculations of such eigenfunctions. Two new solutions, traveling waves and modulated waves (corresponding to periodically reversing blinking states), bifurcate from the standing wave branch in secondary bifurcations, in remarkable qualitative agreement with experiment. These traveling waves are single frequency states traveling predominantly in one or the other direction, but are not rotating waves: there is no comoving frame in which these waves appear steady. The blinking states are quasiperiodic states born in a secondary Hopf bifurcation: the Hopf frequency corresponds to the reversal period. These states persist only for an interval of parameter values, and with increasing forcing give way to (nonreversing) traveling waves, typically via a global bifurcation. More recently, it has been noted that chaotically reversing waves are also possible [19].

The advantage of this approach stems from its relative simplicity: it provides a concise way of modeling the effects of "weakly" breaking the translation symmetry in a system. In fact, the resulting equations can be shown to describe completely the small-amplitude behavior of Cross' amplitude equations [16]. The procedure suggested by Dangelmayr and Knobloch does not, however, address the issue of whether the addition of small, linear, symmetry-breaking terms suffices (a priori) for a complete dynamical description of the effects of distant endwalls.

In this paper we pursue an alternative approach. We 
formulate the problem in a finite container, and then examine the limit as the length of the container becomes large. This formulation requires the use of the standing wave solutions, since, as shown by Dangelmayr and Knobloch $[17,18]$, all the primary bifurcations take this form, a conclusion also reached by Bestehorn, Friedrich, and Haken [20]. In such a formulation, as in the work of Dangelmayr and Knobloch, analogues of traveling waves will be produced through symmetry-breaking secondary bifurcations from such standing wave branches. However, the present approach elucidates the conditions under which the presence of distant endwalls can be considered to be a weak perturbation of the unbounded problem. In particular we show that the correct amplitude equations in the limit of large aspect ratios are described by the normal form for the Hopf bifurcation with $D_{4}$ symmetry in which the $D_{4}$ symmetry is weakly broken. We describe the origin of the $D_{4}$ symmetry and show that Eqs. $(5 \mathrm{a}, \mathrm{b})$ are a special case of our more general equations.

The paper is organized as follows. In Sec. II we discuss properties of Hopf bifurcations in boxes. In Sec. III we obtain finite-dimensional amplitude equations describing the interaction of odd and even parity standing waves, and show that in long boxes these equations have an approximate $D_{4}$ symmetry. In Sec. IV we analyze the resulting equations and in Sec. V complement this analysis with numerical results. Our conclusions are summarized in Sec. VI.

\section{OSCILLATORY CONVECTION IN BOXES}

We begin by considering a general system,

$$
\mathcal{M} \partial_{t} \Psi(x, y, t)=\mathcal{L} \Psi(x, y, t)+\mathcal{N} \Psi(x, y, t),
$$

where $\Psi$ is a multi-component field depending on time $t$ and spatial coordinates $x \in \mathbb{R}, y \in \mathbb{R}^{n-1}$. $\mathcal{M}\left(\partial_{x}, \partial_{y}\right), \mathcal{L}\left(\partial_{x}, \partial_{y}\right)$ are linear partial differential operators, and $\mathcal{N}\left(\partial_{x}, \partial_{y}\right)$ is a nonlinear operator (typically bilinear). We assume that Eqs. (6) are equivariant under the group operations

$$
\begin{aligned}
& x \longrightarrow x+l \quad \text { (translation) } \\
& x \longrightarrow-x \quad \text { (reflection) }
\end{aligned}
$$

but impose endwalls on the system,

$$
B(\Psi)=0 \quad \text { at } x=-L / 2, L / 2 .
$$

The theory that follows requires only that these boundary conditions break the translation invariance of the system while preserving the reflection symmetry $(x \rightarrow-x)$, but is otherwise independent of their detailed form. This is in contrast to the corresponding theory for the onset of the steady state instability in a finite container where the results with Neumann endwall conditions differ, even in the large aspect ratio limit, from the generic result that holds for other types of boundary conditions [21]. This is a consequence of the so-called "hidden" symmetries present in the Neumann case; in the case of the Hopf bifurcation these symmetries are still present but do not introduce any restrictions on the normal form [22].
To understand the effects of such endwalls on the behavior of the system we will consider the limiting case where the endwalls are very far apart ( $L$ large), and compare the results with the unbounded case. On the basis of this comparison we show below that adding distant endwalls to an unbounded system does not represent a simple "perturbation" of the system. In particular, the $L \rightarrow \infty$ and $L=\infty$ limits will not necessarily agree, and fundamentally distinct behaviors will be associated with each.

We denote by $\Phi_{k}(x, y)$ the spatial eigenmodes of the system (6), linearized about the trivial [i.e., $O(2)$ symmetric] equilibrium and subject to the horizontal boundary conditions (8) (along with appropriate boundary conditions for the other spatial directions). Each "mode" is characterized by a particular temporal growth rate. Owing to the boundary conditions at the endwalls, these modes will be quantized, and we index them with $k$. We assume that for some control parameter $R$ below threshold (e.g., a heating rate), all linear modes are exponentially stable. As the control parameter is increased, the modes successively become unstable. This is qualitatively similar to what occurs in the unbounded case, but with two fundamental distinctions:

1. Since the modes of the system are discrete, there will exist a finite gap separating the critical values of the parameter $R$ at which successive modes become unstable. Formally this is due to the discrete nature of the spectrum of the linear stability problem. See Fig. 1. This is in contrast to the case of an unbounded system, where once the critical parameter threshold is crossed, a continuum of unstable modes emerges. Note that the gap spacing approaches zero as the system size $L \rightarrow \infty$; cf. [8]. Nonetheless, such gaps are present for any finite $L$.

2. Once endwalls are placed on an unbounded system, pure traveling wave solutions are no longer possible, regardless of how distant the endwalls are; the large $L$ and

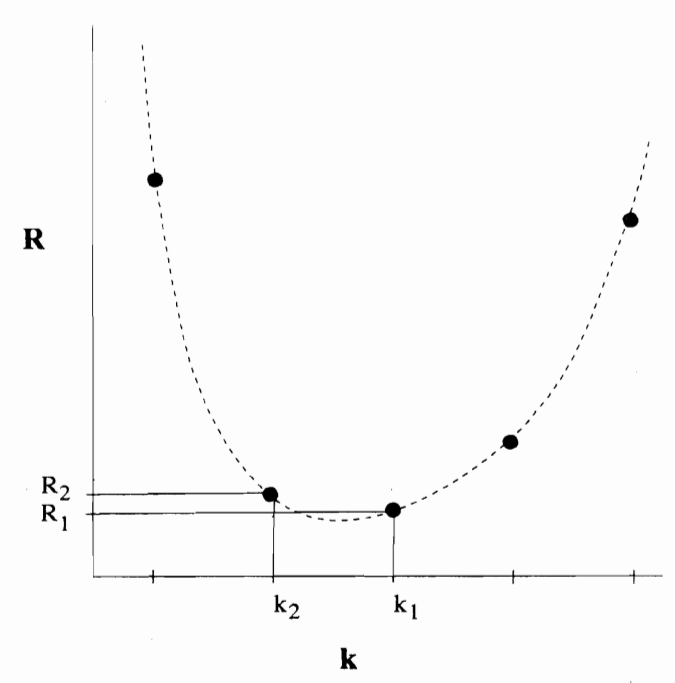

FIG. 1. Rayleigh number vs wave number for the bounded system. 
infinite $L$ cases are fundamentally distinct.

These considerations underlie some of the inherent mathematical difficulties that arise if one attempts to treat distant endwalls as a weak perturbation of the unbounded system. Instead, we begin with the finite problem, and then let the size of the system become large.

Given the set of spatial linear modes $\left\{\Phi_{k}(x, y)\right\}$ (which we assume forms a complete basis), the fields $\Psi(x, y, t)$ can be expanded as

$$
\Psi=\sum_{k} a_{k}(t) \Phi_{k}(x, y)+\bar{a}_{k}(t) \bar{\Phi}_{k}(x, y)
$$

where $a_{k}(t)$ denotes the complex temporal amplitude of the $k$ th spatial mode. Substituting into (6), a (infinite) set of modal amplitude equations is obtained. We are interested in the case of an oscillatory bifurcation, and wish to derive a reduced (finite-dimensional) set of equations that characterizes the behavior of the system in the vicinity of this instability.

We know that at onset $\left(R=R_{1}\right)$ there is a single neutrally stable critical mode $k_{1}$ corresponding to a standing wave pattern; all other modes of the system are stable since they are below their respective thresholds (at $\left.R_{2}, R_{3}, \ldots\right)$. Sufficiently near $R_{1}$ this critical mode can be regarded as the "driving" mode of the system, with all other modes effectively "slaved"; a center manifold reduction could then be performed based on this single critical mode. However, for large aspect ratio systems, such a reduction procedure cannot capture the full physical behavior of the full system (such as the presence of traveling waves). For this purpose the retention of the modes $k_{1}$ and $k_{2}$ (where $k_{2}$ represents the first mode to become unstable after mode $k_{1}$ ) is necessary. As explained below, it is the interaction between these two standing wave modes that produces "traveling" wave behavior, and captures the essential physics of the problem.

The linear spatial modes of the system (6) and (8) can be assumed to be eigenstates of the reflection operator, and hence strictly of even or odd parity. Generically, all such modes set in at simple Hopf bifurcations. Even though in the bounded system the first two (typically, opposite parity [8]) standing wave modes become unstable at slightly different parameter values, for the purposes of a reduction procedure valid in the large $L$ limit, it is crucial to treat these modes as emerging "simultaneously," as though from the degenerate $L=\infty$ case. The resulting mode interaction is capable of describing mixed parity states, such as traveling waves and blinking states. This notion can be formalized by imagining that there exists a second parameter, such as the system length $L$, which can be freely varied. By adjusting this parameter, the first two modes of the bounded system can be arranged to bifurcate simultaneously. A formal center manifold reduction can then be carried out, yielding equations for both critical modes (cf. [23]). Any variation of this second parameter away from its exact value at cocriticality can simply be treated as an unfolding of the bifurcation, and will not effect the dimensionality of the center manifold itself. Alternatively, the device of introducing a second parameter into the problem can be entirely avoided by employing instead a center-unstable manifold reduction
[24] to capture the dynamical behavior of both modes (one mode being slightly unstable when the other is at criticality). In either case in the limit $L \rightarrow \infty$ the normal form coefficients will be independent of $L$ and the resulting unfolding will therefore capture the whole interval of Rayleigh numbers within which only two modes are unstable. At higher Rayleigh numbers, the small-amplitude nonlinear dynamics is often governed by the first one or two modes even though additional modes may also be unstable; cf. [25]. Consequently the applicability of the resulting normal form equations may well extend beyond their formal range of validity.

\section{REDUCTION TO A FINITE-DIMENSIONAL SYSTEM}

In the following we let $z_{1}(t), z_{2}(t)$ denote the complex amplitudes of the first two standing wave modes to go unstable (i.e., the driving modes), and assume $z_{2}$ corresponds to the odd parity mode. A center manifold reduction (or center-unstable manifold reduction) of the full (infinite-dimensional) modal equations in the vicinity of the oscillatory instability will yield a system of coupled equations describing the interaction of these two complex modes. The form of these equations follows from simple considerations. First, since each mode is close to, but not precisely at a Hopf bifurcation, the linearization about the origin for the reduced equations must have the form

$$
\left(\begin{array}{cc}
\mu+i \omega & 0 \\
0 & \mu^{\prime}+i \omega^{\prime}
\end{array}\right)\left(\begin{array}{l}
z_{1} \\
z_{2}
\end{array}\right)
$$

where $\mu, \mu^{\prime}$ are unfolding terms that vanish when a given mode passes through criticality. Second, the nonlinear terms in these equations must be equivariant under $\left(z_{1}, z_{2}\right) \rightarrow\left(z_{1},-z_{2}\right)$, owing to the reflection symmetry $(x \rightarrow-x)$ of the original system (6). A third consideration is slightly more subtle, but crucial. The only obvious symmetry of the center (-unstable) manifold equations is the spatial reflection symmetry $z_{2} \rightarrow-z_{2}$. In fact, however, there exists an additional discrete symmetry hidden in the asymptotic limit: for large $L$, the system has an approximate symmetry under interchange of the two modes,

$$
\left(z_{1}, z_{2}\right) \rightarrow\left(z_{2}, z_{1}\right)
$$

stemming from the fact that these modes are degenerate at $L=\infty$. This symmetry is not exact for any finite $L$. Nonetheless, since it becomes a true symmetry in the large $L$ limit, it will be vital to retain it in the normal form calculations. This interchange property changes the symmetry group of the problem from $Z_{2}$ to $D_{4}$, and thus alters the basic structure of the equations. In particular, this symmetry will force certain coefficients in the normal form equations to vanish, and others to be identical. The fact that this symmetry is only approximate has important consequences and requires the introduction of interchange-breaking terms as unfolding parameters in the interchange-symmetric system. Together, the reflection symmetry and the (approximate) interchange symmetry imply that the reduced equations for $z_{1}, z_{2}$ have 
the form

$$
\begin{aligned}
& \frac{d z_{1}}{d t}=(\mu+i \omega) z_{1}+F\left(z_{1}, \bar{z}_{1}, z_{2}^{2}, z_{2} \bar{z}_{2}, \bar{z}_{2}^{2}\right) \\
& \frac{d z_{2}}{d t}=\left(\mu^{\prime}+i \omega^{\prime}\right) z_{2}+F\left(z_{2}, \bar{z}_{2}, z_{1}^{2}, z_{1} \bar{z}_{1}, \bar{z}_{1}^{2}\right) .
\end{aligned}
$$

We next put the system (12a) and (12b) into normal form, treating the modes $z_{1}, z_{2}$ as bifurcating simultaneously, i.e., $\mu=\mu^{\prime}=0$. This is necessary in order to avoid removing certain formally nonresonant terms; if this is not done the coordinate transformation that removes such terms will develop a singularity in the $L \rightarrow \infty$ limit, and hence will not be acceptable. [Alternatively, this issue can be avoided entirely by adjusting a second system parameter $(L)$ to force $z_{1}, z_{2}$ to become unstable simultaneously.] A related issue concerns the oscillation frequencies $\omega, \omega^{\prime}$ of the two modes. These frequencies also become equal in the large $L$ limit. Hence, as above, to avoid coordinate singularities only those nonresonant terms that remain nonsingular in the $\omega=\omega^{\prime}$ limit should be removed. Once these normal form transformations are completed, the detunings that exist between $\mu, \mu^{\prime}$ and $\omega, \omega^{\prime}$ for finite $L$ will be reintroduced.

The normal form equations are now readily calculated. The linearized equations can be written as

$$
\frac{d \mathbf{z}}{d t}=A \mathbf{z},
$$

where $\mathbf{z}=\left(z_{1}, \bar{z}_{1}, z_{2}, \bar{z}_{2}\right)$ and

$$
A=\left(\begin{array}{cccc}
i \omega & 0 & 0 & 0 \\
0 & -i \omega & 0 & 0 \\
0 & 0 & i \omega & 0 \\
0 & 0 & 0 & -i \omega
\end{array}\right)
$$

Observe that this linearization corresponds to the case of a semisimple double Hopf bifurcation with 1:1 resonance. The nonlinear resonant components of the normal form vector field must commute with

$$
e^{A^{\dagger} \tau}=\left(\begin{array}{cccc}
e^{-i \omega \tau} & 0 & 0 & 0 \\
0 & e^{i \omega \tau} & 0 & 0 \\
0 & 0 & e^{-i \omega \tau} & 0 \\
0 & 0 & 0 & e^{i \omega \tau}
\end{array}\right)
$$

for $\tau \in \mathbb{R}[26]$. An arbitrary monomial term $z_{1}^{p} \bar{z}_{1}^{q} z_{2}^{r} \bar{z}_{2}^{s}$ in the normal form must therefore obey the constraint

$$
p-q+r-s=1 \text {. }
$$

Thus, no quadratic terms will appear. The six possible resonant cubic terms are

$\left|z_{1}\right|^{2} z_{1}, \quad\left|z_{2}\right|^{2} z_{1}, \quad \bar{z}_{1} z_{2}^{2}, \quad\left|z_{2}\right|^{2} z_{2}, \quad\left|z_{1}\right|^{2} z_{2}, \quad \bar{z}_{2} z_{1}^{2}$.

Since the linear part of the vector field (13) and (14) also commutes with the normal form symmetry (15), the full normal form equations will be equivariant under this $S^{1}$ normal form symmetry. The approximate $D_{4}$ symmetry in the problem restricts the way in which the resonant terms appear. In particular, in the $L \rightarrow \infty$ limit, the normal form equations are

$$
\begin{aligned}
& \frac{d z_{1}}{d t}=i \omega z_{1}+A\left|z_{1}\right|^{2} z_{1}+B\left|z_{2}\right|^{2} z_{1}+C \bar{z}_{1} z_{2}^{2}, \\
& \frac{d z_{2}}{d t}=i \omega z_{2}+A\left|z_{2}\right|^{2} z_{2}+B\left|z_{1}\right|^{2} z_{2}+C \bar{z}_{2} z_{1}^{2},
\end{aligned}
$$

where $A, B, C$ are complex coefficients, and the vector field has been truncated at cubic order. Such a truncation is valid subject to appropriate nondegeneracy conditions. One may verify that the system $(18 \mathrm{~b}, \mathrm{~b})$ is equivariant under the group $D_{4} \times S^{1}$ :

$$
\begin{aligned}
D_{4}: & \left(z_{1}, z_{2}\right) \rightarrow\left(z_{1},-z_{2}\right), \\
& \left(z_{1}, z_{2}\right) \rightarrow\left(z_{2}, z_{1}\right), \\
S^{1}: & \left(z_{1}, z_{2}\right) \rightarrow\left(e^{i \theta} z_{1}, e^{i \theta} z_{2}\right) .
\end{aligned}
$$

These equations describe the onset of an oscillatory instability in a box, in the limit that the box length $L$ goes to infinity. Note that this limit, derived on the basis of symmetry considerations (i.e., the group $D_{4}$ ), differs from the normal form equations at $L=\infty$, which is instead described by a Hopf bifurcation with $\mathrm{O}(2)$ symmetry. This observation indicates that the imposition of distant endwalls need not constitute a mild perturbation to the unbounded system.

Equations $(18 \mathrm{a}, \mathrm{b})$ are identical to the equations describing a Hopf bifurcation with $D_{4}$ symmetry considered by Swift [27] in the context of coupled oscillators. However, since our primary interest is in the large but finite $L$ case (for particular scaling regimes), we are led to consider symmetry-breaking unfoldings of this limiting $D_{4}$-symmetric case.

The unfolded equations are easily found: first note that weakly breaking the interchange symmetry (which is only approximate) will not introduce any additional nonlinear terms into the problem. [This is actually a somewhat subtle issue. Only the reflection symmetry $z_{2} \rightarrow-z_{2}$ is an exact symmetry of the problem, yet Eqs. (18a,b) are also equivariant under $z_{1} \rightarrow-z_{1}$. This second discrete symmetry results from the Hopf normal form symmetry (in conjunction with the left/right reflection symmetry), and will be present regardless of whether the interchange symmetry is exact or not. In this study we will not be considering the effects of breaking such normal form symmetries. We also note that for physical systems that have a midplane reflection symmetry in addition to the left/right reflection symmetry, the normal form symmetry $z_{1} \rightarrow-z_{1}$ is exact.] The unfolded equations, through cubic order, are

$$
\frac{d z_{1}}{d t}=(\mu+i \omega) z_{1}+A\left|z_{1}\right|^{2} z_{1}+B\left|z_{2}\right|^{2} z_{1}+C \bar{z}_{1} z_{2}^{2}
$$

$$
\frac{d z_{2}}{d t}=\left(\mu^{\prime}+i \omega^{\prime}\right) z_{2}+A^{\prime}\left|z_{2}\right|^{2} z_{2}+B^{\prime}\left|z_{1}\right|^{2} z_{2}+C^{\prime} \bar{z}_{2} z_{1}^{2}
$$

The unfolding quantities $\mu, \mu^{\prime}, \mu-\mu^{\prime}, \omega-\omega^{\prime}, A-A^{\prime}, B-$ $B^{\prime}, C-C^{\prime}$ are all small. The resulting equations $(20 \mathrm{a}, \mathrm{b})$ describe the interaction of even and odd parity standing waves in the system and will be the main focus for the 
remainder of this paper. These equations should be regarded as describing a double Hopf bifurcation with 1:1 resonance, with the special property that they are close to the $D_{4}$-symmetric problem owing to the large size $L$ of the system. They are exact in the sense that they can be formally derived by the center manifold reduction procedure by requiring that the two modes $z_{1}, z_{2}$ bifurcate simultaneously, and noting that the spectrum corresponding to all other modes remains bounded away from the pure imaginary axis in the complex plane for any finite $L$. We remark that Bestehorn, Friedrich, and Haken [20] have proposed Eqs. (20a,b) as a model for modulated wave behavior in finite boxes. A special case of these equations was studied by Nagata [28].

The crucial observation is that under the coordinate transformation $z_{1}=v+\bar{w}, z_{2}=v-\bar{w}$ (which may be regarded as a transformation from standing wave coordinates to traveling wave coordinates), the system $(20 \mathrm{a}, \mathrm{b})$ becomes identical to the "standard" (Ginzburg-Landaulike) equations $(5 \mathrm{a}, \mathrm{b})$ describing broken translation symmetry, except for the appearance of new cubic terms $\left(\bar{v} \bar{w}^{2}, \bar{w} \bar{v}^{2}\right)$ in $(5 \mathrm{a}, \mathrm{b})$, respectively. Most significantly, the coefficients of these new terms remain $O(1)$ in magnitude even in the limit $L \rightarrow \infty$. This observation demonstrates that such cubic terms must be retained in addition to the small, linear symmetry-breaking terms in order to capture correctly the effects of even distant endwalls.

\section{ANALYSIS OF THE NORMAL FORM EQUATIONS}

\section{A. Scaling considerations}

The asymptotic dynamical behavior of Eqs. $(20 \mathrm{a}, \mathrm{b})$ is determined by the scaling relations that hold among the various unfolding parameters. These scaling relations are set by the following two quantities:

(i) The departure of the control parameter $R$ from its critical values at $R_{1}, R_{2}$ for modes $z_{1}, z_{2}$, respectively. This will determine the growth rates $\mu, \mu^{\prime}$ of the modes.

(ii) The length $L$ of the system. This will set the scale for the differences between the two modes (i.e., $\left.\left|\mu-\mu^{\prime}\right|,\left|\omega-\omega^{\prime}\right|,\left|A-A^{\prime}\right|,\left|B-B^{\prime}\right|,\left|C-C^{\prime}\right|\right)$. At times it will prove useful to treat the system length $L$ itself as a control parameter of the system, which can be used to modify the generic scaling behavior of $\left|\mu-\mu^{\prime}\right|$.

We determine first the various scaling relations among the unfolding parameters set by the system length $L$. Let $R_{c}(m, L)$ denote the critical value of the parameter $R$ at which the $m$ th mode first becomes unstable. In general the critical parameter values $R_{c}$ will be functions of the quantity $m / L$, i.e., $R_{c}=R_{c}(m / L)$, in order that in the limit $L \rightarrow \infty$, the minimum value of $R_{c}$ (over all possible $m$ ) should remain $O(1)$ in magnitude, as in the infinite case. [The quantity $m / L$ can be regarded as the dominant wave number of a mode. For large $L$ systems, the modes that first go unstable generate wave patterns whose local length scale is similar to that of the unbounded case, i.e., $O(1)$.] Since the complex amplitudes $z_{1}, z_{2}$ in $(20 \mathrm{a}, \mathrm{b})$ represent the first two modes of the system to become unstable, the (integer) mode numbers corresponding to these modes will (usually) differ in magnitude by one, and we denote them by $M, M+1$. These two integers yield the minimum values of $R_{c}(m / L)$ for all integers $m$. Letting $R_{1}=R_{c}(M / L), R_{2}=R_{c}[(M+1) / L]$ be the critical values for the two modes, it follows that

$$
\left|R_{1}-R_{2}\right| \sim O\left(1 / L^{2}\right) .
$$

This derives from the fact that $R_{1}, R_{2}$ lie near the minimum of the "curve" $R_{c}=R_{c}(m / L)$; in other words $M, M+1 \approx M^{*}$, where $M<M^{*}<M+1$ and the (noninteger) $M^{*}$ satisfies

$$
\left.\frac{d R_{c}(m / L)}{d m}\right|_{M^{*}}=0
$$

Since $m$ and $L$ appear as a ratio, the scaling result (21) follows. This difference in the critical values for the two modes, $\left|R_{1}-R_{2}\right|$, sets the scaling behavior for the difference in linear growth rates $\left|\mu-\mu^{\prime}\right|$ in the normal form equations $(20 \mathrm{a}, \mathrm{b})$. Note that by making small [i.e., $O(1)$ ] adjustments in the system length $L$, the two modes $M, M+1$ can be made to bifurcate simultaneously, thereby reducing the deviation $\left|\mu-\mu^{\prime}\right|$ below $O\left(1 / L^{2}\right)$. This mechanism is responsible for the sensitive dependence of the resulting behavior on the aspect ratio.

We next consider how the difference in oscillation frequencies between the two modes scales with $L$. As a function of $m$ and $L$, the oscillation frequency of a given mode may be written as $\Omega=\Omega(m / L)$. This follows from the requirement that the frequency of the modes $M, M+1$ should remain $O(1)$ as $L \rightarrow \infty$. Letting $\Omega_{1}=\Omega(M / L)$, $\Omega_{2}=\Omega[(M+1) / L]$, the frequency deviation between the critical modes is

$$
\left|\Omega_{1}-\Omega_{2}\right| \sim O(1 / L) .
$$

The quantity $\left|\Omega_{1}-\Omega_{2}\right|$ sets the scaling behavior of $\left|\omega-\omega^{\prime}\right|$ in Eqs. (20a,b). Similar reasoning to that above shows that the quantitites $\left|A-A^{\prime}\right|,\left|B-B^{\prime}\right|,\left|C-C^{\prime}\right|$ also scale as $1 / L$ in the limit of large $L$.

The final scaling relation in the normal form equations, giving the magnitude of the growth rates $\mu, \mu^{\prime}$, is determined by the deviation of the parameter $R$ from its critical values at $R_{1}, R_{2}$ for the two modes. More precisely, the average growth rate $\left(\mu+\mu^{\prime}\right) / 2$ in $(20 \mathrm{a}, \mathrm{b})$ scales with $R-\Delta R$, where $\Delta R \equiv\left(R_{1}+R_{2}\right) / 2$.

There is no intrinsic scaling relation between the scalesetting parameters $\Delta R$ and $L$, and a variety of choices is available. However, only for one of these do the normal form equations $(20 \mathrm{a}, \mathrm{b})$ provide (generically) an asymptotically well-defined description of the behavior in the original system (6). A second scaling choice, corresponding to a more restrictive set of conditions, will also yield exact asymptotic equations, as will be described later.

\section{B. Asymptotic scaling: $\Delta R \sim 1 / L^{2}, \Delta \omega \sim 1 / L$}

This scaling of the growth rate with aspect ratio is a "natural" one, and it is the one assumed implicitly in various other reduction schemes appearing in the litera- 
ture (e.g., [13]). This derives from the fact that as $R$ is raised above its minimum critical value, the number of spatial modes activated (i.e., unstable) in the system is

$$
\begin{aligned}
N & \sim \frac{\sqrt{R-R_{c}}}{\text { wave number spacing between adjacent modes }} \\
& \sim L \sqrt{\Delta R} .
\end{aligned}
$$

Thus, for the scaling $\Delta R \sim 1 / L^{2}$, the number of active modes is $O(1)$. As $\Delta R$ is lowered towards its critical value, a point is reached below which there are only two active modes left - these correspond to the two critical amplitudes $z_{1}, z_{2}$ in $(20 \mathrm{a}, \mathrm{b})$. Hence, for this choice of scaling of $\Delta R$ with $L$, the normal form equations have a well-defined limit. Evidently this two-mode analysis formally breaks down if $\Delta R$ is increased too much. However, even in this case, the normal form equations $(20 \mathrm{a}, \mathrm{b})$ might still provide a reasonable (though incomplete) description of the basic dynamics.

To analyze this scaling limit, we rewrite equations $(20 \mathrm{a}, \mathrm{b})$ in a slightly more useful form:

$$
\begin{aligned}
\frac{d z_{1}}{d t}= & {[(\mu+\Delta \mu)+i(\omega+\Delta \omega)] z_{1}+[A+\Delta A]\left|z_{1}\right|^{2} z_{1} } \\
& +[B+\Delta B]\left|z_{2}\right|^{2} z_{1}+[C+\Delta C] \bar{z}_{1} z_{2}^{2}, \\
\frac{d z_{2}}{d t}= & {[(\mu-\Delta \mu)+i(\omega-\Delta \omega)] z_{2}+[A-\Delta A]\left|z_{2}\right|^{2} z_{2} } \\
& +[B-\Delta B]\left|z_{1}\right|^{2} z_{2}+[C-\Delta C] \bar{z}_{2} z_{1}^{2} .
\end{aligned}
$$

Observe that the $O(1)$ frequency $\omega$ can be omitted, since it can be pulled from the equations via the redefinition $z_{1} \rightarrow e^{i \omega t} z_{1}, z_{2} \rightarrow e^{i \omega t} z_{2}$. In what follows we assume this has been done. We define $\epsilon$ to be our scaling parameter $\left(L \sim 1 / \epsilon, \Delta R \sim \epsilon^{2}\right)$, setting

$$
\begin{aligned}
\mu & =\epsilon^{2} \hat{\mu}, & & z_{1}=\epsilon v_{1}+\epsilon^{2} v_{2}, \\
\Delta \mu & =\epsilon^{2} \Delta \hat{\mu}, & & z_{2}=\epsilon w_{1}+\epsilon^{2} w_{2}, \\
\Delta \omega & =\epsilon \Delta \hat{\omega}, & & \partial_{t}=\epsilon \partial_{t_{1}}+\epsilon^{2} \partial_{t_{2}} .
\end{aligned}
$$

Substituting, and dropping the carets, the leading order terms in $\epsilon$ yield

$$
\begin{aligned}
{\left[\partial_{t_{1}}-i \Delta \omega\right] v_{1} } & =0 \\
{\left[\partial_{t_{1}}+i \Delta \omega\right] w_{1} } & =0 .
\end{aligned}
$$

These equations are readily solved. We find

$$
v_{1}=P\left(t_{2}\right) e^{i \Delta \omega t_{1}}, \quad w_{1}=Q\left(t_{2}\right) e^{-i \Delta \omega t_{1}}
$$

where $P, Q$ are arbitrary functions of the slow time scale $t_{2}$. At next order in $\epsilon$ we have

$$
\begin{aligned}
{\left[\partial_{t_{1}}-i \Delta \omega\right] v_{2}=} & -\partial_{t_{2}} v_{1}+(\mu+\Delta \mu) v_{1}+A\left|v_{1}\right|^{2} v_{1} \\
& +B\left|w_{1}\right|^{2} v_{1}+C \bar{v}_{1} w_{1}^{2} \\
{\left[\partial_{t_{1}}+i \Delta \omega\right] w_{2}=} & -\partial_{t_{2}} w_{1}+(\mu-\Delta \mu) w_{1}+A\left|w_{1}\right|^{2} w_{1} \\
& +B\left|v_{1}\right|^{2} w_{1}+C \bar{w}_{1} v_{1}^{2} .
\end{aligned}
$$

Substituting in from (27), the solvability conditions are

$$
\partial_{t_{2}} P=(\mu+\Delta \mu) P+A|P|^{2} P+B|Q|^{2} P,
$$

$$
\partial_{t_{2}} Q=(\mu-\Delta \mu) Q+A|Q|^{2} Q+B|P|^{2} Q,
$$

where we have used the fact that terms that go as $e^{i \Delta \omega t_{1}}$ in Eq. (28a) are resonant, as are $e^{-i \Delta \omega t_{1}}$ terms in (28b).

The amplitude equations $(29 \mathrm{a}, \mathrm{b})$ provide an asymptotically exact description near the onset of the oscillatory instability. Comparing these equations with the normal form equations $(24 a, b)$, it is clear that the primary effect of the asymptotic scaling (25) is to eliminate the terms $\bar{z}_{1} z_{2}^{2}, \bar{z}_{2} z_{1}^{2}$ in (24a) and $(24 \mathrm{~b})$, respectively. This is because the frequency difference $(\Delta \omega)$ between the modes is much larger than the difference in growth rates $(\Delta \mu)$, i.e., $1 / L$ versus $1 / L^{2}$. Consequently the behavior of each mode on the "fast" time scale is dominated by its oscillation frequency, i.e., $z_{1} \sim e^{i \Delta \omega t}, z_{2} \sim e^{-i \Delta \omega t}$; cf. (27). Looking now at the nonlinear terms in $(24 \mathrm{a}, \mathrm{b})$ it is clear that the final term in each fluctuates rapidly compared to the first two terms and hence effectively "averages out" to zero. Even in the absence of these terms, however, the resulting equations continue to differ from "standard" equations $(5 a, b)$ by the presence of additional cubic terms, as described previously.

The emergence of this "fast" time scale associated with $\Delta \omega$ can be understood rather simply, and has a natural analog in the unbounded problem. Consider the two spatial modes $z_{1}$ and $z_{2}$. These will be characterized by slightly different length scales, and hence slightly different wave numbers. The unfolding parameter $\Delta \omega$ measures the variation of the standing wave oscillation frequency with wave number. In the case of an unbounded system, it can be naturally associated with the group velocity of the traveling wave solutions. Hence there exists a "fast" time scale in the unbounded case as well. However, in the standard derivation of the coupled complex Ginzburg-Landau equations for an oscillatory instability in an unbounded system, one demands that the time scale associated with the group velocity be slow, even though this will only be true under very special circumstances, e.g., near the codimension-two point. Though this problem has been recognized, the correct Ginzburg-Landau equations have been derived only recently [12] and only in the unbounded case. These new equations are integrodifferential in nature, and are more complex than the standard Ginzburg-Landau equations. It is therefore rather remarkable that for bounded systems, this same fast time scale leads to a simplified set of equations $(29 a, b)$.

As a result of this averaging over a fast time scale, the amplitude equations $(29 \mathrm{a}, \mathrm{b})$ possess an extra phase shift symmetry, and are readily analyzed. Letting $P=r_{1} e^{i \theta_{1}}$, $Q=r_{2} e^{i \theta_{2}}$ yields equations of the form [29]

$$
\begin{aligned}
\frac{d r_{1}}{d t} & =(\mu+\Delta \mu) r_{1}+\left(A_{r} r_{1}^{2}+B_{r} r_{2}^{2}\right) r_{1}, \\
\frac{d r_{2}}{d t} & =(\mu-\Delta \mu) r_{2}+\left(A_{r} r_{2}^{2}+B_{r} r_{1}^{2}\right) r_{2}, \\
\frac{d \theta_{1}}{d t} & =A_{i} r_{1}^{2}+B_{i} r_{2}^{2}, \\
\frac{d \theta_{2}}{d t} & =A_{i} r_{2}^{2}+B_{i} r_{1}^{2},
\end{aligned}
$$

where $A_{r}, B_{r}, A_{i}, B_{i}$ denote the real and imaginary parts of the coefficients. We distinguish four types of solutions: 
1. The trivial state $\left(r_{1}, r_{2}\right)=(0,0)$. This solution corresponds to the no motion (conducting) state of a system. It is stable if $0<|\Delta \mu|<-\mu$, a saddle if $|\Delta \mu|>|\mu|$, and a source if $0<|\Delta \mu|<\mu$.

2. A pure mode $\left(r_{1}, r_{2}\right)=\left(r_{1}, 0\right)$. This solution at $r_{1}=\sqrt{-(\Delta \mu+\mu) / A_{r}}$ corresponds to a pure (single frequency) standing wave (SW1) in the original system. It exists if either (1) $A_{r}>0, \Delta \mu<-\mu$, or (2) $A_{r}<0, \Delta \mu>-\mu$. For both cases, one eigenvalue is $\left[\left(A_{r}-B_{r}\right) \mu-\left(A_{r}+B_{r}\right) \Delta \mu\right] / A_{r}$; the other eigenvalue is strictly positive for case (1), and strictly negative for case (2).

3. A pure mode $\left(r_{1}, r_{2}\right)=\left(0, r_{2}\right)$. This second standing wave (SW2) solution at $r_{2}=\sqrt{(\Delta \mu-\mu) / A_{r}}$ exists if either (1) $A_{r}>0, \Delta \mu>\mu$, or (2) $A_{r}<0, \Delta \mu<\mu$. For both cases, one eigenvalue is $\left[\left(A_{r}-B_{r}\right) \mu+\left(A_{r}+\right.\right.$ $\left.\left.B_{r}\right) \Delta \mu\right] / A_{r}$; the other eigenvalue is strictly positive for case (1), and strictly negative for case (2).

4. Mixed mode fixed point. This fixed point in $\left(r_{1}, r_{2}\right)$ space satisfies

$$
\left(\begin{array}{c}
r_{1}^{2} \\
r_{2}^{2}
\end{array}\right)=\frac{1}{B_{r}^{2}-A_{r}^{2}}\left(\begin{array}{c}
\left(A_{r}-B_{r}\right) \mu+\left(A_{r}+B_{r}\right) \Delta \mu \\
\left(A_{r}-B_{r}\right) \mu-\left(A_{r}+B_{r}\right) \Delta \mu
\end{array}\right)
$$

and represents a two-frequency modulated wave (MW), consisting of a mixture of the even and odd parity modes $z_{1}, z_{2}$. If

$$
0<\left|A_{r}\right|<B_{r}, \quad \mu<0, \quad|\Delta \mu|<\frac{\left(A_{r}-B_{r}\right)}{\left(A_{r}+B_{r}\right)} \mu
$$

or if

$$
0<\left|A_{r}\right|<-B_{r}, \quad \mu>0, \quad|\Delta \mu|<-\frac{\left(A_{r}-B_{r}\right)}{\left(A_{r}+B_{r}\right)} \mu,
$$

then the modulated wave exists and is a saddle. If

$$
0<\left|B_{r}\right|<A_{r}, \quad \mu<0, \quad|\Delta \mu|<-\frac{\left(A_{r}-B_{r}\right)}{\left(A_{r}+B_{r}\right)} \mu,
$$

the modulated wave solution is a source. It is a sink if

$$
0<\left|B_{r}\right|<-A_{r}, \quad \mu>0, \quad|\Delta \mu|<\frac{\left(A_{r}-B_{r}\right)}{\left(A_{r}+B_{r}\right)} \mu .
$$

We summarize these results in the following bifurcation diagrams [Figs. $2(\mathrm{a})-2(\mathrm{~d})]$ in the $(\mu, \Delta \mu)$ plane. For each figure, the $(\mu, \Delta \mu)$ plane divides into six regions. The boundaries are defined by the lines: $\Gamma_{0}: \mu+\Delta \mu=0, \Gamma_{1}$ : $\mu-\Delta \mu=0$, and the half lines: $\Gamma_{2}: \Delta \mu=\frac{1+B_{r}}{1-B_{r}} \mu, \Gamma_{3}:$ $\Delta \mu=\frac{B_{r}+1}{B_{r}-1} \mu$. Within each of the six regions is drawn the associated $\left(r_{1}, r_{2}\right)$-phase portrait. (Note that, through rescalings and parameter symmetries, we have restricted to the case $A_{r}=-1$ with no loss in generality.) Observe that the MW solutions bifurcate from the SW1, SW2 branches in a pitchfork bifurcation in the reduced $\left(r_{1}, r_{2}\right)$ space.

It is interesting to consider the temperature eigenfunction corresponding to these various solutions. Recall that the eigenfunction associated with modes $z_{1}, z_{2}$ in Eqs. $(20 a, b)$ is of the form

$$
\begin{aligned}
\vartheta(x, y, t) \sim & \mathbb{R}\left\{\left[z_{1}(t) F_{e}(x)+z_{2}(t) F_{o}(x)\right] f(y)\right\} \\
& +(\text { higher-order terms }),
\end{aligned}
$$

where $F_{e}, F_{o}$ are the even and odd parity horizontal spatial eigenfunctions. For illustrative purposes, we take [16]

$$
\begin{aligned}
& F_{e}(x)=\left\{e^{-\gamma x+i k x}+e^{\gamma x-i k x}\right\} \cos \frac{\pi x}{L} \\
& F_{o}(x)=\left\{e^{-\gamma x+i k x}-e^{\gamma x-i k x}\right\} \cos \frac{\pi x}{L}
\end{aligned}
$$

defined on the domain $\left[-\frac{L}{2}, \frac{L}{2}\right]$. Here $\gamma=(\alpha+i \beta) / L$ is a complex parameter depending on the group velocity of free traveling waves, and $k$ is their wave number. This choice of eigenfunctions is in excellent qualitative agreement with the form of the primary unstable modes as determined from experiments (see Kolodner [7]). For this reason, we will use this functional form to illustrate the various possible solutions to Eqs. $(24 \mathrm{a}, \mathrm{b})$. For the pure standing wave solutions, we set $\left(z_{1}, z_{2}\right)=\left(r_{1} e^{i \omega t}, 0\right)$, $\left(0, r_{2} e^{i \omega^{\prime} t}\right)$, while for the modulated wave, we write $\left(z_{1}, z_{2}\right)=\left(r_{1} e^{i \omega t}, r_{2} e^{i \omega^{\prime} t}\right)$. The former [Figs. 3(a),(b)] takes the form of a chevron pattern, consisting of waves propagating in opposite directions in the two halves of the container. Note that we refer to such patterns as standing waves because they are pure parity states. In contrast, the latter tends to be localized first in one half of the box, and at time $O(L)$ later, in the other half of the box. This behavior is illustrated in Fig. 4. The sloshing back and forth of the modulated wave qualitatively resembles the "blinking state" behavior observed in binary fluid and doubly diffusive convection experiments $([3,4])$.

It is important to note that by varying the aspect ratio $L$ by a small [i.e., $O(1)$ ] amount, different regions of the $(\mu, \Delta \mu)$ plane can be accessed. Hence, the theory predicts that the type of behavior found near onset will be highly sensitive to aspect ratio, as observed experimentally [3].

\section{Asymptotic scaling: $\Delta R \sim 1 / L^{2}, \Delta \omega \sim 1 / L^{2}$}

We next consider the original scaling relation $\Delta R \sim$ $1 / L^{2}$ and supplement it with the auxiliary condition $\Delta \omega \sim 1 / L^{2}$. A multiscale asymptotic analysis similar to that described above now recovers the full normal form equations $(24 \mathrm{a}, \mathrm{b})$ (neglecting deviations in the nonlinear coefficients), i.e., there is no longer any "averaging out" of any terms in the equations. This is because Eqs. (24a,b) are in fact invariant under the rescaling: $\mu \rightarrow \epsilon^{2} \mu, \Delta \mu \rightarrow \epsilon^{2} \Delta \mu, \Delta \omega \rightarrow \epsilon^{2} \omega, z_{1} \rightarrow \epsilon z_{1}, z_{2} \rightarrow \epsilon z_{2}$, $t \rightarrow t / \epsilon^{2}$. Hence, with this new scaling, Eqs. $(24 \mathrm{a}, \mathrm{b})$ also provide an asymptotically well-defined description for the underlying system. The requirement $\Delta \omega \sim 1 / L^{2}$ implies that the difference in oscillation frequencies between the two modes is smaller than what it would be under generic circumstances. This can be achieved by adjusting a second control parameter in the system and is entirely analogous to the (implicit) assumption made in the usual derivation of the Ginzburg-Landau equations that the group velocity of a traveling wave is small. With 


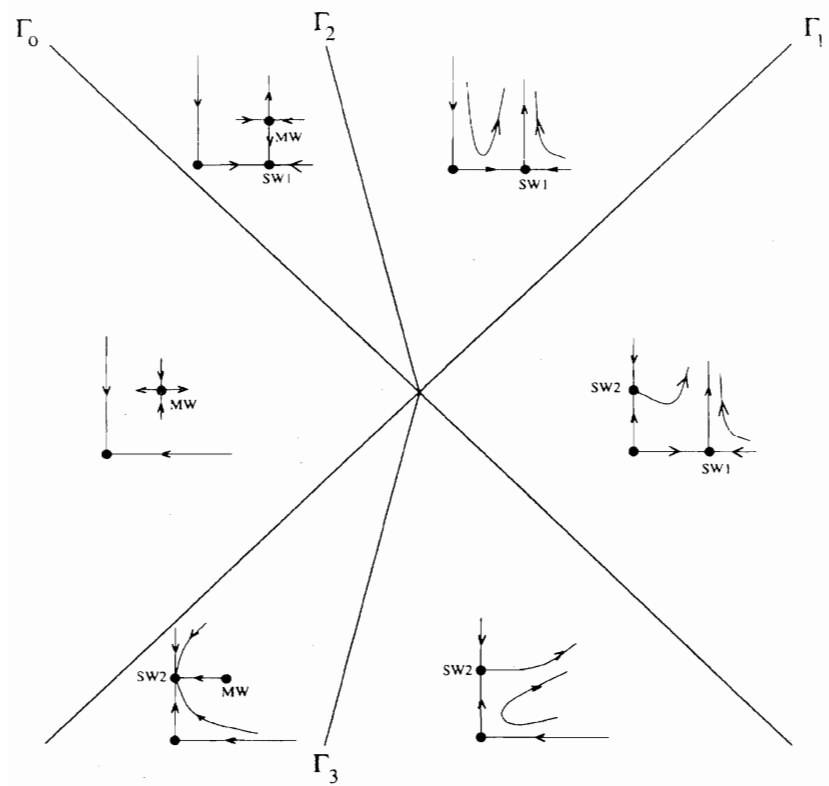

$\underline{B_{r}>1}$

(a)

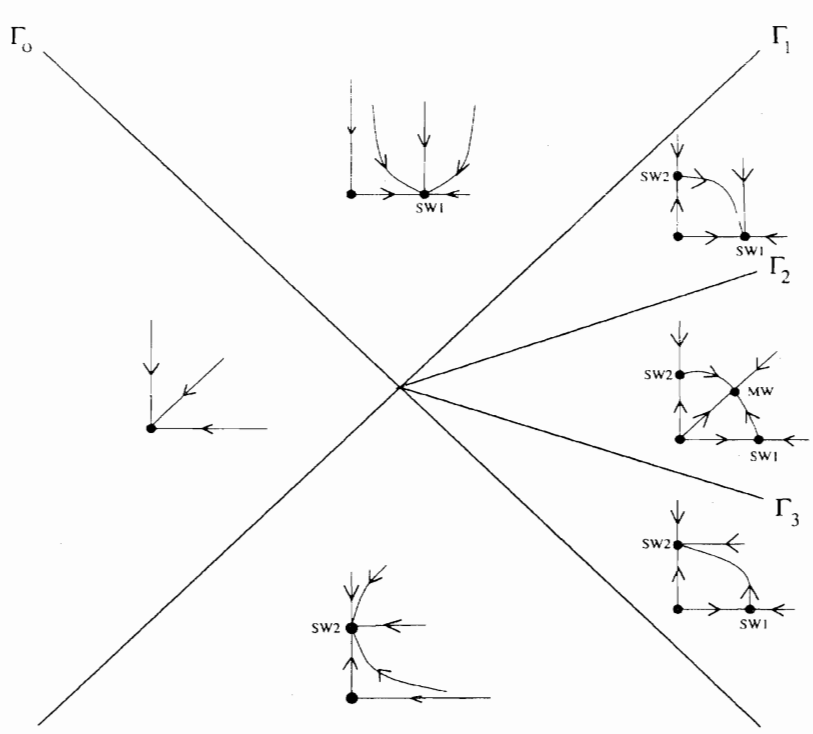

$-1<B_{r}<0$

(c)

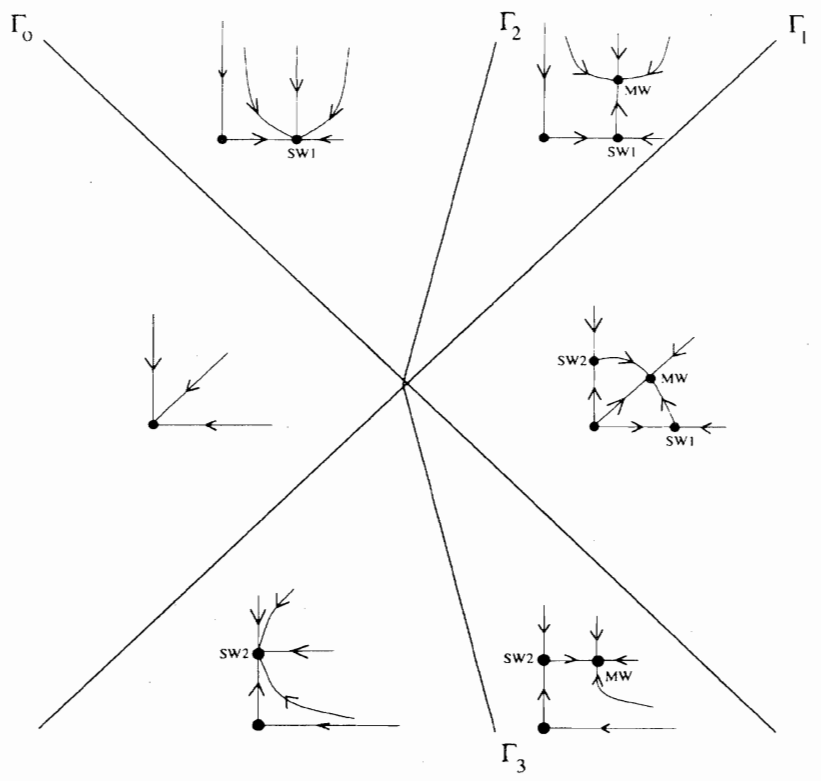

$\underline{0<B_{r}<1}$

(b)

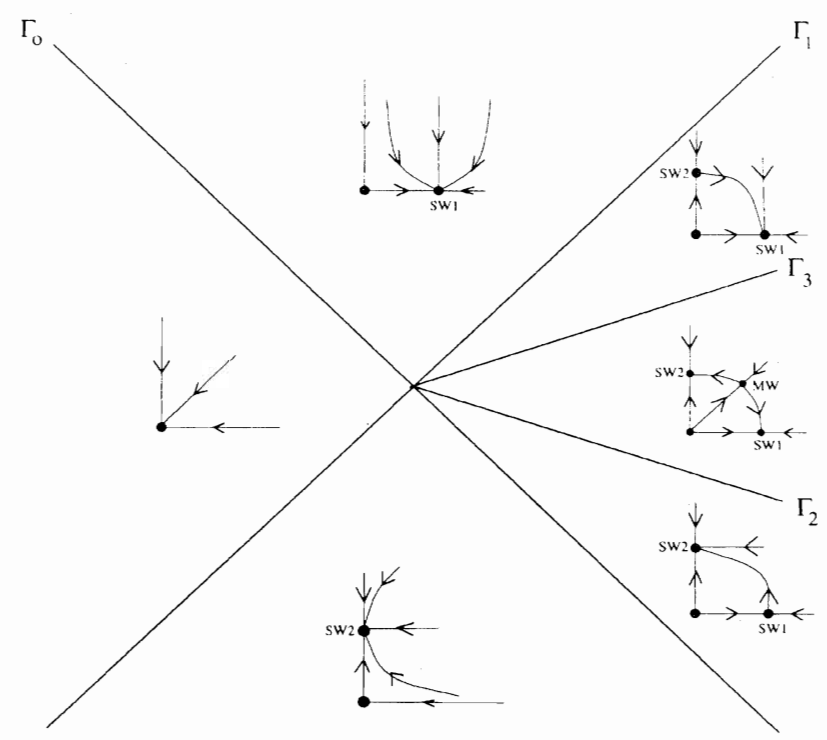

$\underline{B_{r}<-1}$

(d)

FIG. 2. The bifurcation diagrams for $A_{r}=-1$ and (a) $B_{r}>1$, (b) $0<B_{r}<1$, (c) $-1<B_{r}<0$, and (d) $B_{r}<-1$. 
this scaling there is no longer a fast time scale in the system and thus certain terms in the normal form equations no longer average to zero.

The analysis of the normal form equations $(24 \mathrm{a}, \mathrm{b})$ divides naturally into two parts, the Hopf bifurcation with $D_{4}$ symmetry when the parameters $\Delta \mu, \Delta \omega, \Delta A, \Delta B, \Delta C$ vanish, and the nonsymmetric case when they are small but nonzero. We begin by reviewing the $D_{4}$-symmetric problem.

\section{The $D_{4}$-symmetric case}

In the $D_{4}$-symmetric case Eqs. $(24 \mathrm{a}, \mathrm{b})$ have the symmetry

$$
\left(z_{1}, z_{2}\right) \rightarrow\left(z_{1},-z_{2}\right), \quad\left(z_{1}, z_{2}\right) \rightarrow\left(z_{2}, z_{1}\right),
$$

in addition to the $S^{1}$ normal form symmetry. To study these equations, the following coordinate transformation proves useful [27]:

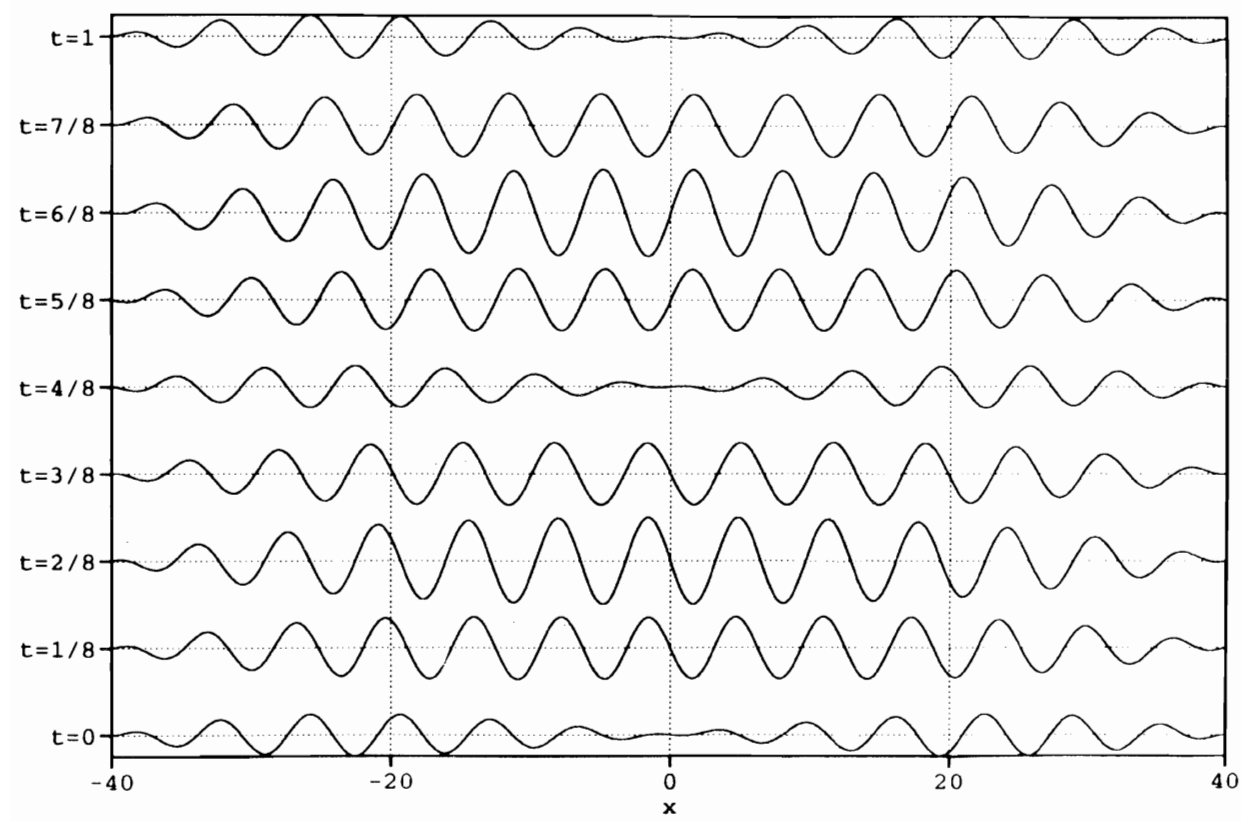

(a)

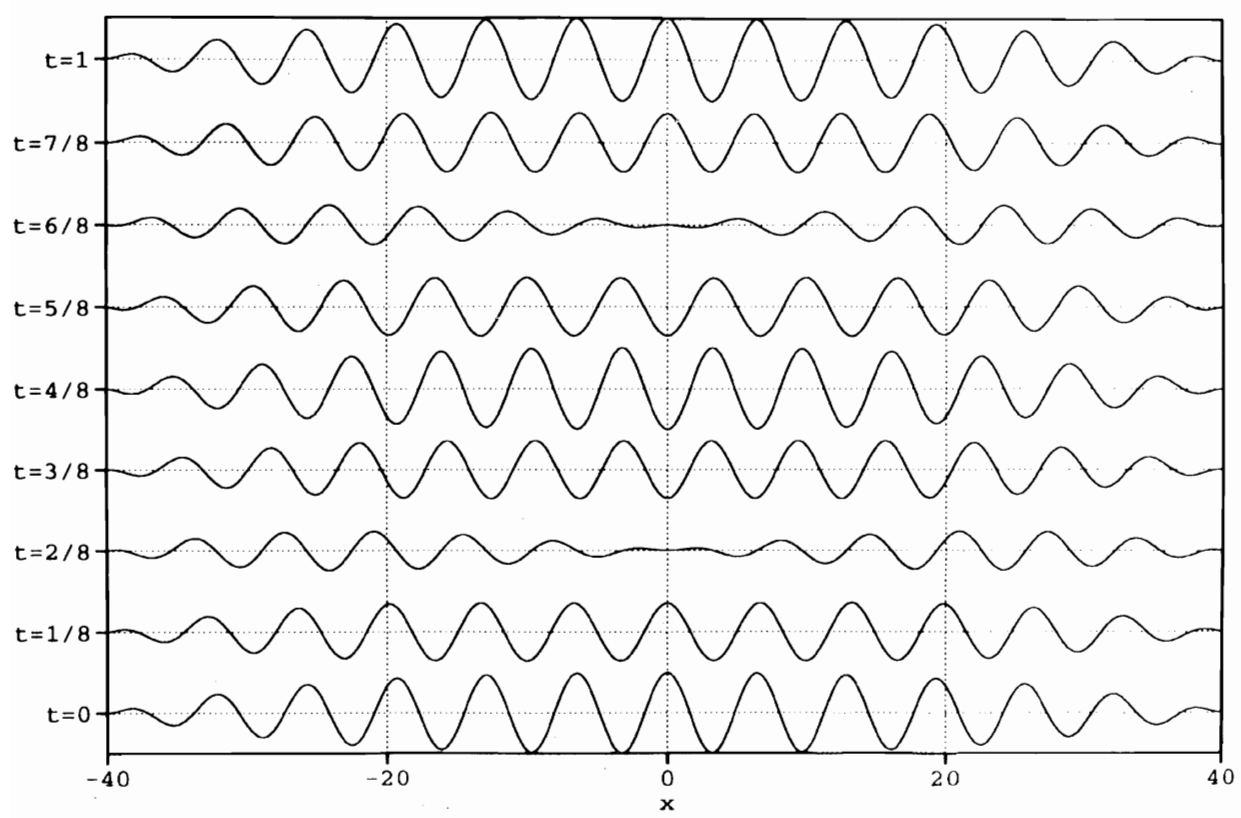

FIG. 3. Odd and even parity states $\vartheta\left(x, y_{0}, t\right)$ given by $(32)$ and $(33 a, b)$ as a function of $x \in\left(-\frac{L}{2}, \frac{L}{2}\right)$, at a sequence of times separated by $\Delta t=\frac{1}{8} \frac{2 \pi}{\omega}$, for $L=80, \omega\left(\approx \omega^{\prime}\right)=2 \pi$, $k=1, \alpha=2.5, \beta=2.0$. 


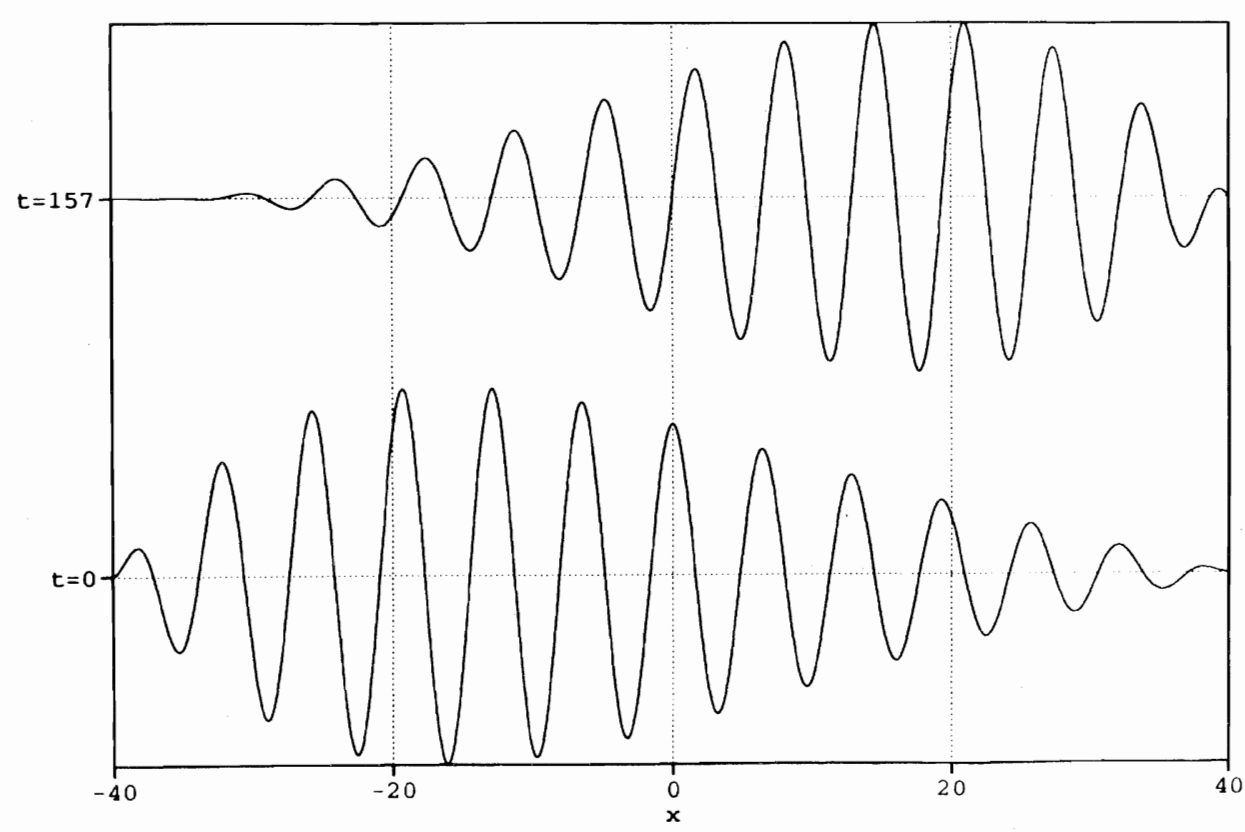

FIG. 4. A modulated wave shown at two successive times $(t=0,157.0)$ for $\omega=6.293$, $\omega^{\prime}=6.273, r_{1}=1.6, r_{2}=1.3$, $L=80, k=1, \alpha=2.5$, $\beta=2.0$.

$$
\begin{aligned}
u+i v & =r \sin \theta e^{i \phi}=2 z_{1} \bar{z}_{2}, \\
w & =r \cos \theta=\left|z_{1}\right|^{2}-\left|z_{2}\right|^{2}, \\
e^{i \psi} & =\frac{z_{1} z_{2}}{\left|z_{1} z_{2}\right|} .
\end{aligned}
$$

In terms of the $(u, v, w, \psi)$ coordinates, Eqs. $(24 \mathrm{a}, \mathrm{b})$ take the form

$$
\begin{aligned}
& \frac{1}{2} \frac{d u}{d t}=u\left(\mu+R_{u} r\right)+\left(I_{v}-I_{w}\right) v w \\
& \frac{1}{2} \frac{d v}{d t}=v\left(\mu+R_{v} r\right)+\left(I_{w}-I_{u}\right) w u \\
& \frac{1}{2} \frac{d w}{d t}=w\left(\mu+R_{w} r\right)+\left(I_{u}-I_{v}\right) u v
\end{aligned}
$$

where $r=\sqrt{u^{2}+v^{2}+w^{2}}>0$, along with a decoupled equation for the phase $\psi$. The parameters $R_{u}, R_{v}, R_{w}, I_{u}, I_{v}, I_{w}$ are defined in terms of the coefficients $A, B, C$. by

The symmetry of the reduced system $(36 \mathrm{a}-\mathrm{c})$ is given

$$
\begin{aligned}
& \kappa_{1}:(u, v, w) \rightarrow(-u,-v, w) \\
& \kappa_{2}:(u, v, w) \rightarrow(u,-v,-w),
\end{aligned}
$$

constituting the group $Z_{2} \times Z_{2} \approx D_{2}$. There exist three symmetric fixed points of the form $(u, 0,0),(0, v, 0),(0,0, w)$, which represent periodic orbits in the four-dimensional phase space of the $D_{4}$-symmetric system. A nonsymmetric fixed point $(u, v, w)$ also exists for certain parameter regions, and also corresponds to single-frequency solutions in the original system. There is no chaos in this system because the dynamics takes place on a two-dimensional manifold. To see this we write the $D_{4}$-symmetric equations in terms of the coordinates $(r, \theta, \phi)$ defined in $(35 \mathrm{a}-\mathrm{c})$,

$$
\begin{aligned}
\frac{d r}{d t}= & r\left(2 \mu+r\left\{A_{r}+B_{r}+\left[\left(A_{r}-B_{r}\right) \cos ^{2} \theta\right]\right.\right. \\
& \left.\left.+C_{r} \sin ^{2} \theta \cos 2 \phi\right\}\right), \\
\frac{d \theta}{d t}= & r \sin \theta\left[\cos \theta\left(B_{r}-A_{r}+C_{r} \cos 2 \phi\right)-C_{i} \sin 2 \phi\right],
\end{aligned}
$$

$$
\frac{d \phi}{d t}=r\left[\cos \theta\left(A_{i}-B_{i}-C_{i} \cos 2 \phi\right)-C_{r} \sin 2 \phi\right]
$$

where $A_{r}, B_{r}, C_{r}, A_{i}, B_{i}, C_{i}$ denote the real and imaginary parts of the coefficients $A, B, C$, and note that the quantity $\frac{d \theta}{d \phi}$ is independent of $r$. Consequently, one can think of projecting the three-dimensional system $(r, \theta, \phi)$ onto the two-dimensional "associated spherical system" $(\theta, \phi)[27]$. Under appropriate restrictions, there exists a one-to-one correspondence between the fixed points and limit cycles in the associated spherical system, and those in the three-dimensional system $(38 \mathrm{a}-\mathrm{c})$. By analyzing the behavior within the two-dimensional system, Swift finds that when the nonsymmetric fixed points are absent and all the symmetric fixed points bifurcate supercritically and are unstable, a stable limit cycle must be present. This limit cycle appears to be unique up to symmetry, and can take the form of either a "libration" or a "rotation" depending on parameters [30]. Such limit cycles represent two-frequency solutions in the original (four-dimensional) normal form equations, and are the analogues of the two-frequency modulated waves considered earlier. These two types of limit cycles are separated by a homoclinic orbit. Heteroclinic connections can be found in the special case $\mu=R_{u}=R_{v}=R_{w}=0$ when equations $(36 \mathrm{a}-\mathrm{c})$ reduce to the Euler equations, 
and when $R_{u}=R_{v}$. These connections are prime candidates for generating chaotic dynamics once the $D_{4}$ symmetry is broken.

\section{The nonsymmetric case}

We now consider the full normal form equations $(24 \mathrm{a}, \mathrm{b})$, for which the $\mathrm{D}_{4}$ symmetry is only approximate, corresponding to a large but finite container. We begin by reducing these normal form equations to a threedimensional system using the $(u, v, w)$ coordinates introduced in $(35 \mathrm{a}-\mathrm{c})$ :

$$
\begin{aligned}
\frac{1}{2} \frac{d u}{d t}= & \mu u+R_{u} r u+\left(I_{v}-I_{w}\right) v w-\Delta \omega v-\Delta I_{v} r v \\
& +\left(\Delta R_{w}-\Delta R_{u}\right) w u, \\
\frac{1}{2} \frac{d v}{d t}= & \mu v+R_{v} r v+\left(I_{w}-I_{u}\right) w u+\Delta \omega u+\Delta I_{u} r u \\
& +\left(\Delta R_{w}-\Delta R_{v}\right) w v, \\
\frac{1}{2} \frac{d w}{d t}= & \mu w+R_{w} r w+\left(I_{u}-I_{v}\right) u v+\Delta \mu r+\Delta R_{u} u^{2} \\
& +\Delta R_{v} v^{2}+\Delta R_{w} w^{2} .
\end{aligned}
$$

Here

$$
\begin{aligned}
R_{u}+i I_{u} & =(A+B+C) / 2, \\
R_{v}+i I_{v} & =(A+B-C) / 2, \\
R_{w}+i I_{w} & =A, \\
\Delta R_{u}+i \Delta I_{u} & =(\Delta A+\Delta B+\Delta C) / 2, \\
\Delta R_{v}+i \Delta I_{v} & =(\Delta A+\Delta B-\Delta C) / 2, \\
\Delta R_{w}+i \Delta I_{w} & =\Delta A .
\end{aligned}
$$

$$
\left(\begin{array}{ccc}
2\left(\mu+R_{u} r\right) & -2 \Delta \omega-(1+2 \alpha) w & 0 \\
2 \Delta \omega+(-1+2 \alpha) w & 2\left(\mu+R_{v} r\right) & 0 \\
0 & 0 & 2\left(\mu+\Delta \mu \frac{w}{r}+2 R_{w} r\right)
\end{array}\right)
$$

For the even parity standing wave fixed point $w_{e}$, the eigenvalue associated with the $w$ direction is $-2(\mu+\Delta \mu)$. Hence $w_{e}$ is stable in the $w$ direction for $R_{w}<0$ and unstable for $R_{w}>0$. Similarly, for the (odd parity) $w_{o}$ solution, the eigenvalue is $-2(\mu-\Delta \mu)$, and the fixed point is stable in the $w$ direction for $R_{w}<0$, unstable for $R_{w}>0$. The even mode undergoes a $\kappa_{1}$-breaking steady state bifurcation at

$$
\begin{aligned}
{\left[(\Delta \omega)^{2}+\mu^{2}\right]-} & {\left[2 \alpha \Delta \omega+\mu\left(R_{u}+R_{v}\right)\right] \frac{\mu+\Delta \mu}{R_{w}} } \\
+ & {\left[\alpha^{2}-\frac{1}{4}+R_{u} R_{v}\right] \frac{(\mu+\Delta \mu)^{2}}{R_{w}^{2}}=0 . }
\end{aligned}
$$

The corresponding condition for the odd mode is obtained by setting $\Delta \mu \rightarrow-\Delta \mu, \Delta \omega \rightarrow-\Delta \omega$ in (45). Such (pitchfork) bifurcations will give rise to nonsymmetric fixed points, which correspond to single-frequency, traveling wave solutions in the original system. Except very close to where they first branch from the standing
Note that these equations are equivariant under

$$
\kappa_{1}:(u, v, w) \rightarrow(-u,-v, w) ;
$$

The $\kappa_{2}$ symmetry (37) associated with the $D_{4}$-symmetric case is no longer present.

In the following we set

$\Delta R_{u}=\Delta R_{v}=\Delta R_{w}=\Delta I_{u}=\Delta I_{v}=\Delta I_{w}=0$,

as indicated by the present scaling. Only in cases where certain degenerate structures are present will it be necessary to retain these terms. Moreover, through rescalings we may also set $I_{v}-I_{w}=-\frac{1}{2}-\alpha, I_{w}-I_{u}=-\frac{1}{2}+\alpha$, $I_{u}-I_{v}=1$, while absorbing the factors of $1 / 2$ on the left-hand side of $(39 a-c)$ into a redefined time scale. The pure modes $\left(z_{1}, 0\right)$ and $\left(0, z_{2}\right)$ now take the form

$$
\begin{gathered}
(u, v, w)=\left(0,0,-\frac{\mu+\Delta \mu}{R_{w}}\right) \text { if }-\frac{\mu+\Delta \mu}{R_{w}}>0 \\
(u, v, w)=\left(0,0, \frac{\mu-\Delta \mu}{R_{w}}\right) \text { if } \frac{\mu-\Delta \mu}{R_{w}}<0
\end{gathered}
$$

These two solutions, which we call $w_{e}, w_{o}$ for short, represent the even and odd parity standing wave modes in the original system. Note that the fact that $w_{o}$ is invariant under the reflection (41) is an artifact of the coordinate transformation $(35 \mathrm{a}-\mathrm{c})$ leading to the reduced equations $(39 \mathrm{a}-\mathrm{c})$; in the original coordinates $w_{o}$ is odd under parity.

Owing to the reflection symmetry (41) in the problem, the line $w=$ const is invariant under the flow. Consequently, the Jacobian determinant governing the stability of these fixed points factors as follows:

wave solutions, these fixed points cannot be found analytically without additional scaling assumptions. We will therefore study these solutions and others numerically.

\section{NUMERICAL RESULTS}

We have performed a series of numerical simulations on the normal form equations $(20 a, b)$ and various equivalent representations [e.g., Eqs. $(39 \mathrm{a}-\mathrm{c})]$. These numerical studies were carried out using a DSTOOL simulation package, as well as a fourth-order, variable-step Runge-Kutta scheme in MATHEMATICA.

\section{A. Standing and traveling waves}

Since Eqs. $(39 \mathrm{a}-\mathrm{c})$ are a perturbation of the $D_{4}$ symmetric normal form, it is helpful to first provide a physical interpretation for the various possible fixed points of Eqs. $(36 \mathrm{a}-\mathrm{c})$. The fixed points of the form $(0,0, w)$ correspond to the primary standing wave modes of the system with either odd $(w<0)$ or even $(w>0)$ parity. Specifically, using the representation $(32),(33 a, b)$, 


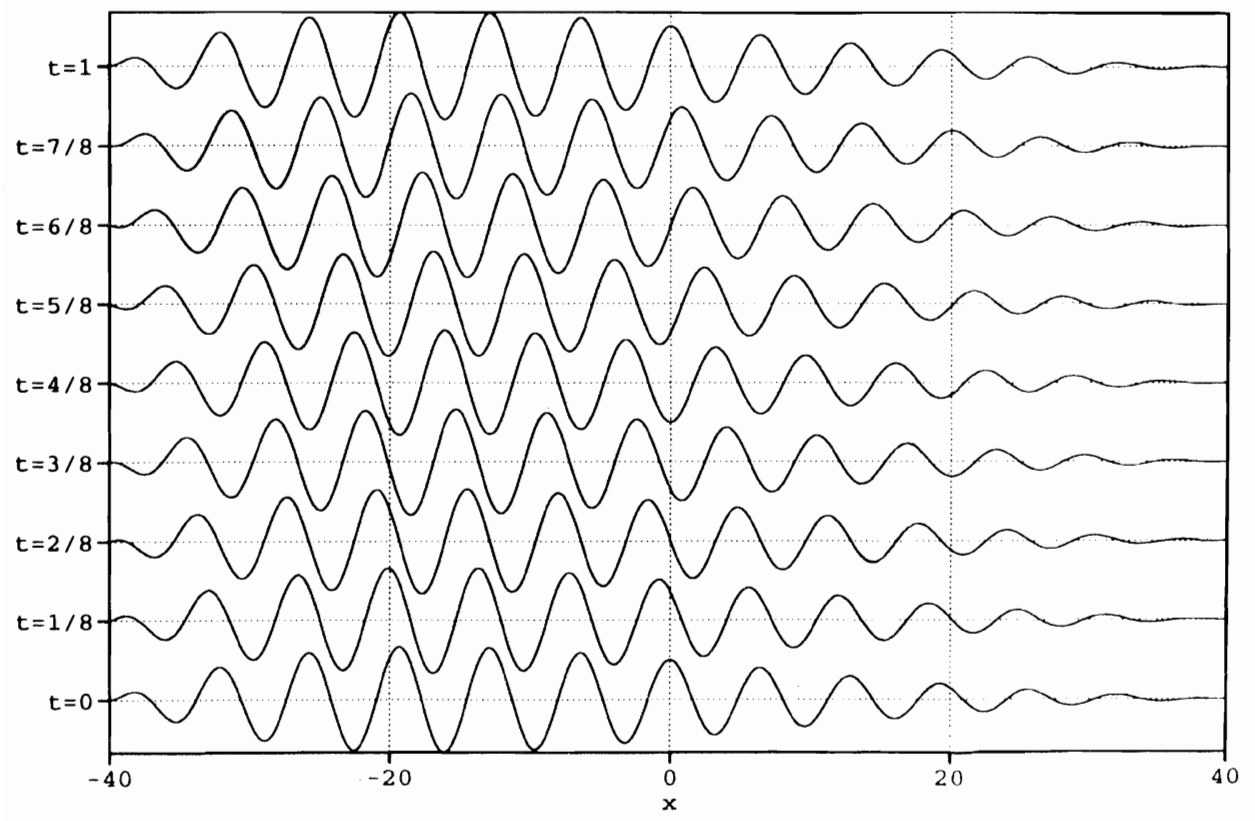

(a)

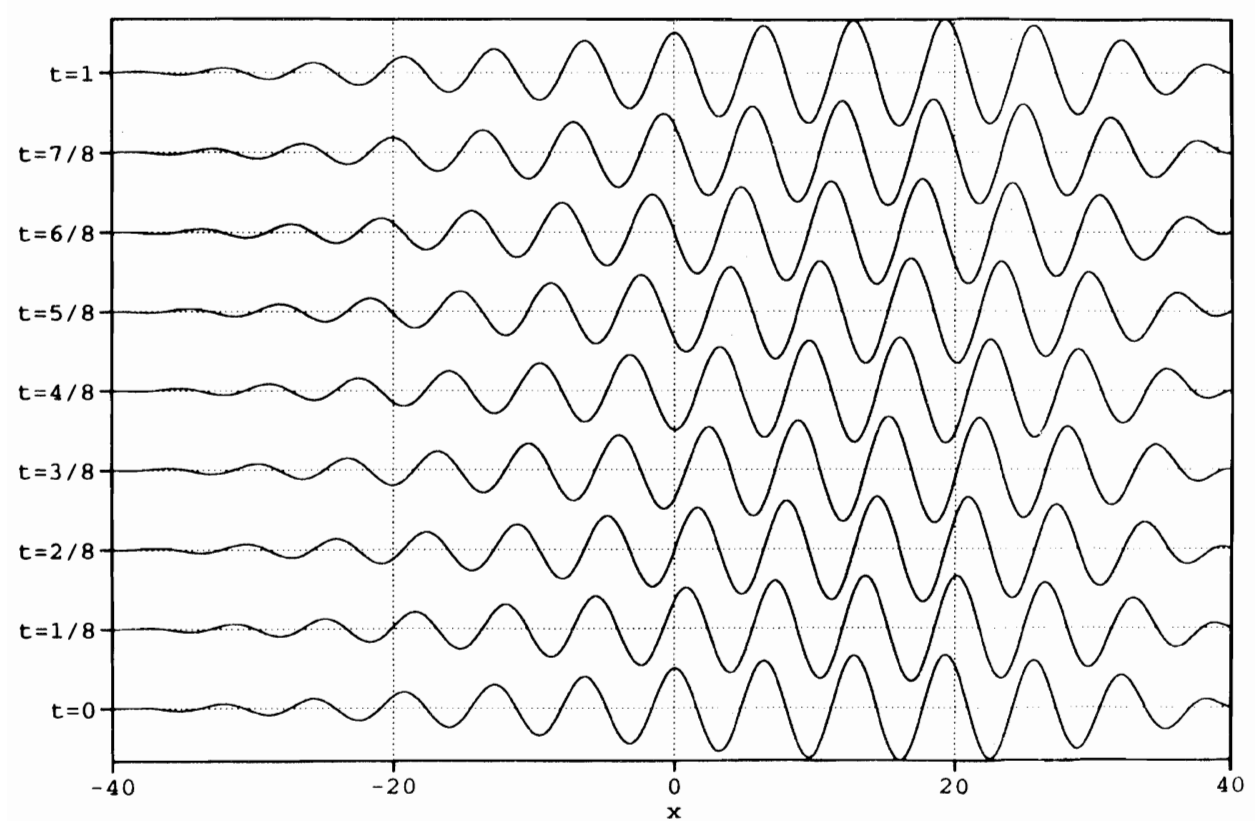

(b)
FIG. 5. (a), (b) Traveling wave states of the form (47) and (c), (d) mixed parity states of the form (48). The solutions are periodic in time. The plots are constructed using the numerical parameter values given in Fig. 3.

$$
\vartheta(x, y, t)=\sqrt{\mp w}\left\{e^{-\alpha x / L} \cos \left(\omega t+k x-\frac{\beta x}{L}\right) \mp e^{\alpha x / L} \cos \left(\omega t-k x+\frac{\beta x}{L}\right)\right\} \cos \frac{\pi x}{L} f(y)
$$

These solutions are illustrated in Figs. 3(a,b). The fixed points $(u, 0,0)$ are either left-traveling waves localized near the left wall $(u>0)$ or right-traveling waves localized near the right wall $(u<0)$ :

$$
\vartheta(x, y, t)=2 \sqrt{ \pm u} e^{\mp \alpha x / L} \cos \left(\omega t \pm k x \mp \frac{\beta x}{L}\right) \cos \frac{\pi x}{L} f(y)
$$

See Figs. $5(\mathrm{a}, \mathrm{b})$. Finally, fixed points of the form $(0, v, 0)$ represent a type of mixed parity standing wave mode: 


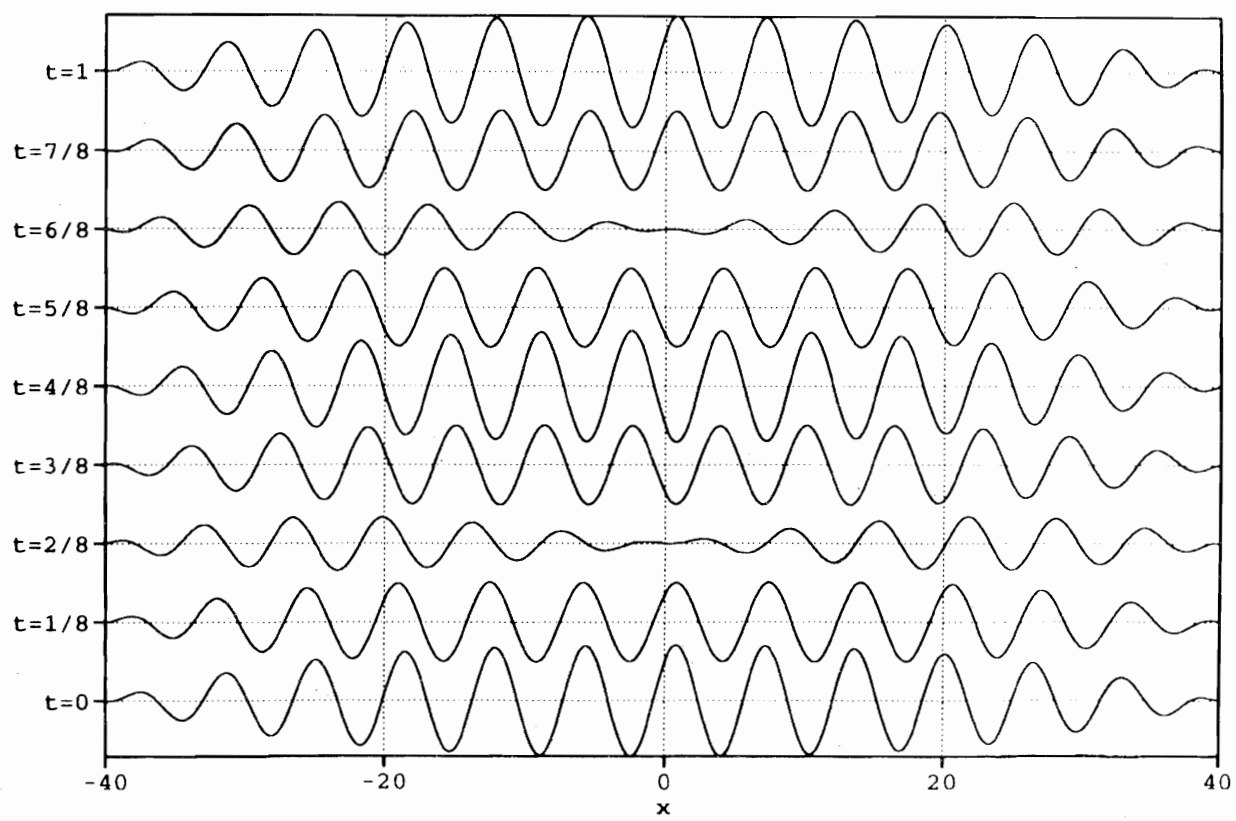

(c)

FIG. 5 (Continued).

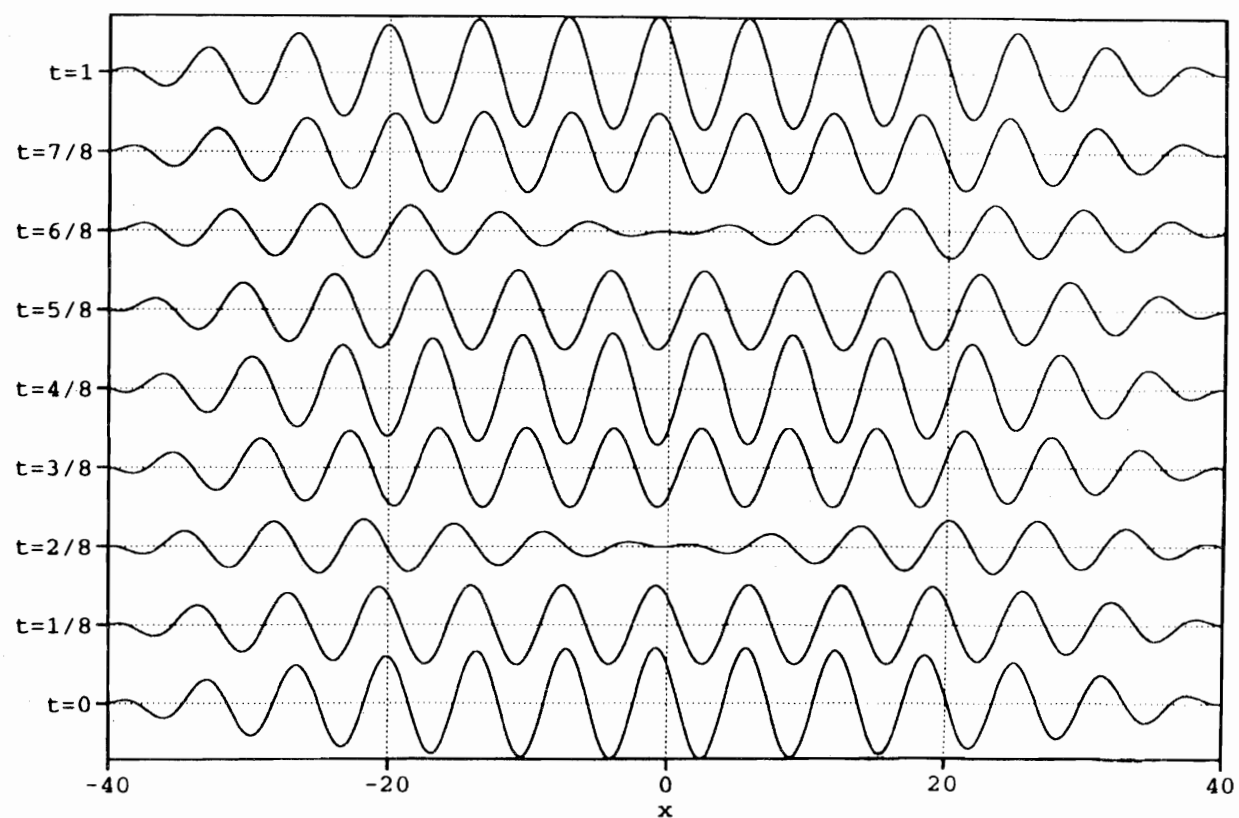

(d)

$$
\vartheta(x, y, t)=\sqrt{ \pm 2 v}\left\{e^{-\alpha x / L} \cos \left(\omega t+k x-\frac{\beta x}{L} \mp \frac{\pi}{4}\right)+e^{\alpha x / L} \cos \left(\omega t-k x+\frac{\beta x}{L} \pm \frac{\pi}{4}\right)\right\} \cos \frac{\pi x}{L} f(y)
$$

See Figs. 5(c,d). Note that there is no spatiotemporal symmetry that relates the solution for $v>0$ with that for $v<0$. This is because these two solutions are related by the symmetry $\kappa_{2}$, which is an interchange symmetry between the odd and even parity modes. The existence of this mixed parity state in the perturbed problem was noted in a related context by Nagata [28].

The perturbed equations $(39 a-c)$ have fixed points that are either fixed by the remaining reflection $\kappa_{1}$ or that break it. The symmetric fixed points continue to be of the form $(0,0, w)$ and are thus standing waves of either odd or even parity, while the nonsymmetric fixed points 
take the general form $(u, v, w)$. The latter can look like perturbations of localized traveling waves if $v, w$ are both small, or the mixed parity waves if $u, w$ are small. More generally the nonsymmetric fixed points combine characteristics of all three types of waves, with a gradual transformation of one type into another as parameters are varied. All of these patterns break reflection symmetry and hence exhibit some propagative features. We will therefore refer to the nonsymmetric fixed points collectively as traveling waves. All are singly periodic in time. Numerically we find that the nonsymmetric fixed points emerge either by bifurcating from a standing wave fixed point [in a steady state (pitchfork) bifurcation], or through a saddle-node bifurcation. We illustrate these possibilities in Fig. 6.
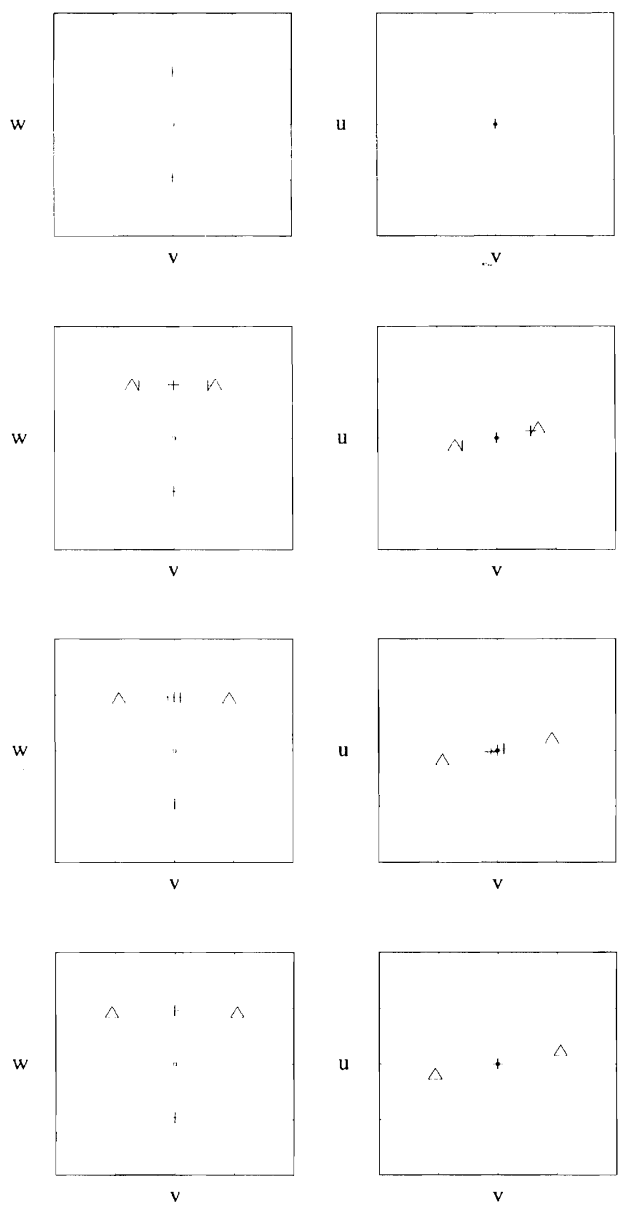

FIG. 6. Typical fixed point structures in the reduced space $(u, v, w)$ for equations (39a-c), shown for increasing values of the parameter $\mu$. Projections in the $(v, w)$ and $(v, u)$ planes are given. Triangles denote sinks, crosses denote saddles. The standing wave solutions lie along the line $u=v=0$. Observe that the traveling wave solutions can emerge either through a saddle-node bifurcation, or through a steady state bifurcation from the standing wave branch.

\section{B. Modulated waves and global bifurcations}

If a symmetric or nonsymmetric fixed point $(u, v, w)$ undergoes a supercritical Hopf bifurcation, a stable, periodic orbit emerges. In the full four-dimensional phase space, this solution represents a two-frequency (quasiperiodic) modulated wave. Modulated waves may be classified as symmetric or asymmetric, according to whether they remain invariant under the symmetry operation $(u, v, w) \rightarrow(-u,-v, w)$ of the system $(39 \mathrm{c}-\mathrm{c})$. We identify the symmetric modulated waves with symmetric blinking states, and the nonsymmetric ones with
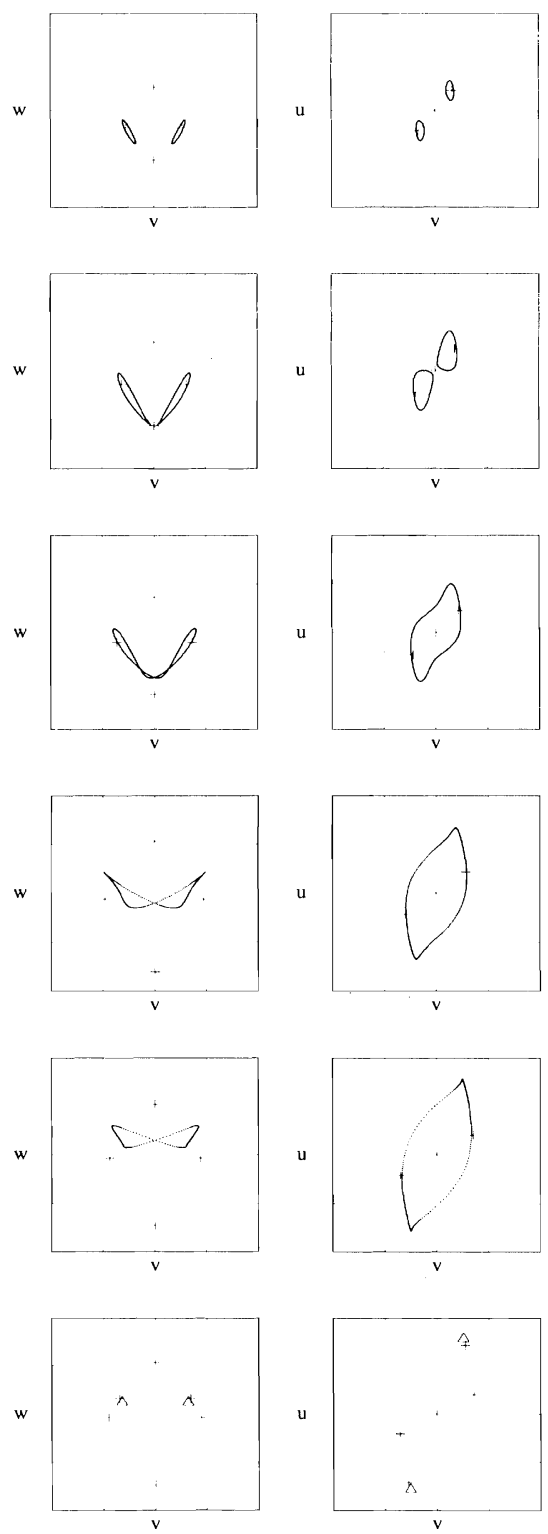

FIG. 7. Phase portraits for equations (39a-c) with $\Delta \mu=$ $-0.0082, \Delta \omega=0.02, R_{u}=-1, R_{v}=-3, R_{w}=-2, \alpha=$ 1.3 , showing a series of global bifurcations for the modulated waves as the parameter $\mu$ is increased from 0.0225 to 0.051 . 
oscillations about a confined traveling wave. Both solution types have been identified by Cross [15] in the Ginzburg-Landau model and are also present in the system $(5 \mathrm{a}, \mathrm{b})[16,19]$. The symmetric solutions were also noted by Bestehorn, Friedrich, and Haken [20]. We have numerically tracked both solution types away from onset, and found that they can undergo an interesting assortment of heteroclinic bifurcations. A representative bifurcation sequence is illustrated in Fig. 7 as the parameter $\mu$ is varied. Projections onto the $(v, w)$ and $(v, u)$ planes are given. The sequence shows a pair of asymmetric (small-amplitude) modulated waves born in a Hopf bifurcation from an asymmetric traveling wave pair. The orbits grow in amplitude, and combine in a (symmetryincreasing) gluing bifurcation to form a symmetric modulated wave solution. This symmetric orbit continues to grow, spending an increasing amount of time near the "cusps" (see the figures for $\mu=0.05$ ). Such cusps are due to "ghost" fixed points. A heteroclinic connection forms near $\mu=0.051$ as two new pairs of nonsymmetric fixed points (formerly the "ghost points") come into being via a saddle-node bifurcation. One member of each pair is stable (denoted by the triangle in the last set of figures), and these stable traveling waves attract all nearby trajectories. This transition thus corresponds to the usual picture of frequency locking.

It is interesting to compare the global bifurcations present in the normal form equations $(39 a-c)$ with those found in the $D_{4}$-symmetric case. Recall that Swift [27] located heteroclinic connections involving various fixed points for the $D_{4}$-symmetric problem. For that case, it was not crucial which pairs of fixed points were involved, since all pairs of points could be related via a parameter symmetry of the problem. However, for the purposes of comparing such bifurcations with those in our full (non- $D_{4}$-symmetric) normal form equations, it is necessary to distinguish two cases, namely, bifurcations that, in the $D_{4}$-symmetric case, are heteroclinic to the fixed points of the form $( \pm u, 0,0)$ and those heteroclinic to the fixed points $(0,0, \pm w)$. The motivation for this is as follows: as noted earlier [cf. 37] the symmetry of the " $D_{4}$-symmetric" problem in the reduced phase space coordinates $(u, v, w)$ is generated by the reflections

$$
\begin{aligned}
& \kappa_{1}:(u, v, w) \rightarrow(-u,-v, w) ; \\
& \kappa_{2}:(u, v, w) \rightarrow(u,-v,-w) .
\end{aligned}
$$

The fixed points $(0,0, \pm w)$ are invariant under $\kappa_{1}$, while the fixed points $( \pm u, 0,0)$ break the $\kappa_{1}$ symmetry, being instead invariant under $\kappa_{2}$. The full (nonsymmetric) normal form equations $(39 \mathrm{a}-\mathrm{c})(\Delta \mu, \Delta \omega \neq 0)$ destroy the $\kappa_{2}$ symmetry of the problem but preserve the $\kappa_{1}$ equivariance. Hence $\kappa_{1}$-symmetric fixed points of the form $(0,0, \pm w)$ continue to exist (i.e., the standing waves), while those that break the $\kappa_{1}$ symmetry are now associated with (asymmetric) traveling wave solutions. Thus, qualitatively different global bifurcations occur in the full normal form equations depending upon whether the corresponding heteroclinic bifurcation in the $D_{4}$-symmetric case is to $\kappa_{1}$-symmetric or $\kappa_{1}$-breaking fixed points. [The remaining case of the $(0, \pm v, 0)$ fixed points is analogous to the $( \pm u, 0,0)$ case.]

We begin with the global bifurcations involving the standing wave fixed points. In the $D_{4}$-symmetric problem $(\Delta \mu=\Delta \omega=0)$ we have a pair of periodic orbits (i.e., the modulated waves) that are invariant under $\kappa_{2}$. (The two members of the pair are related to one another by the symmetry $\kappa_{1}$.) These orbits grow in amplitude as the parameter $R_{u}$ is varied. They then join (at $R_{u}=-2$ ) in a heteroclinic connection involving the two fixed points $(0,0, \pm w)$ (i.e., the two opposite-parity standing waves). This connection then breaks, and a pair of periodic orbits emerges that breaks the $\kappa_{2}$ symmetry. This new pair is invariant under $\kappa_{1} \kappa_{2}$. This global bifurcation thus induces a $\kappa_{2} \rightarrow \kappa_{1} \kappa_{2}$ symmetry exchange in the modulated waves, the bifurcation being mediated by a pair of standing wave solutions. This is illustrated in Fig. 8. All modulated waves considered here are stable.

In contrast, when the $D_{4}$ symmetry of the normal form equations is weakly broken $(\Delta \mu=0.005, \Delta \omega=0.0003)$,
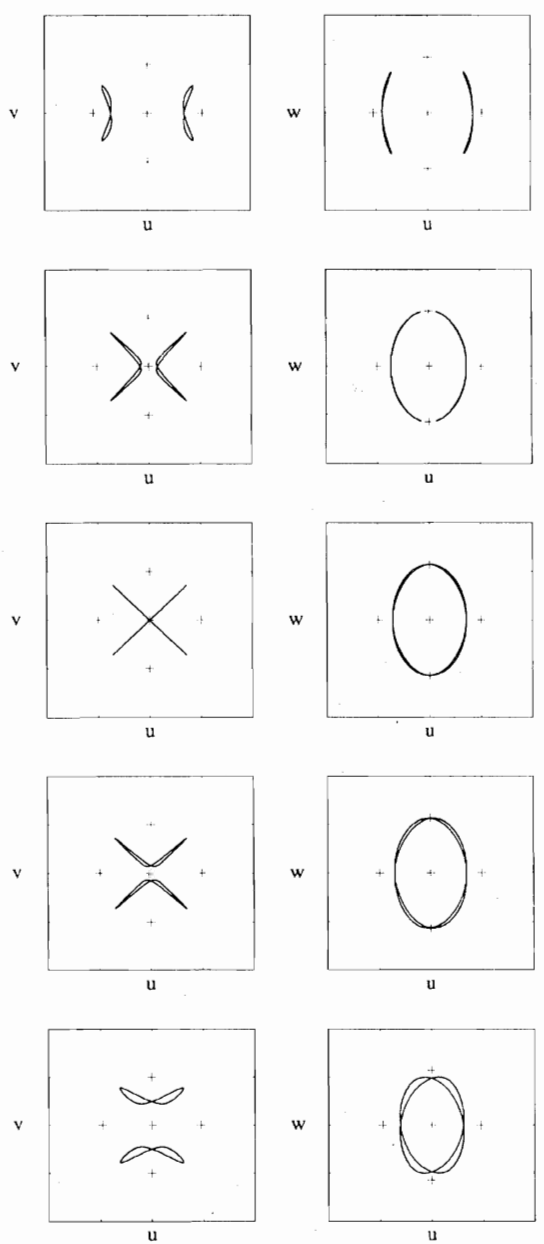

FIG. 8. Modulated waves for the $D_{4}$-symmetric case, with $\mu=0.05, \Delta \mu=0, \Delta \omega=0, R_{v}=-2, R_{w}=-1.75, \alpha=0$. The sequence shows the projections onto the $(u, v)$ and $(u, w)$ planes as the parameter $R_{u}$ is varied from -1.9 to -2.1 . 
the initial pair of modulated waves is no longer $\kappa_{2}$ invariant, although the two waves remain related to one another under $\kappa_{1}$. As $R_{u}$ is varied, these solutions eventually merge and form a single, $\kappa_{1}-$ symmetric orbit. This orbit then pinches off in an "ungluing" bifurcation involving a single homoclinic point at $(0,0,-w)$. Following this homoclinic bifurcation to a standing wave, a pair of new periodic orbits emerges, both of which again break the $\kappa_{1}$ symmetry. This is illustrated in Fig. 9.

We next consider global bifurcations of modulated
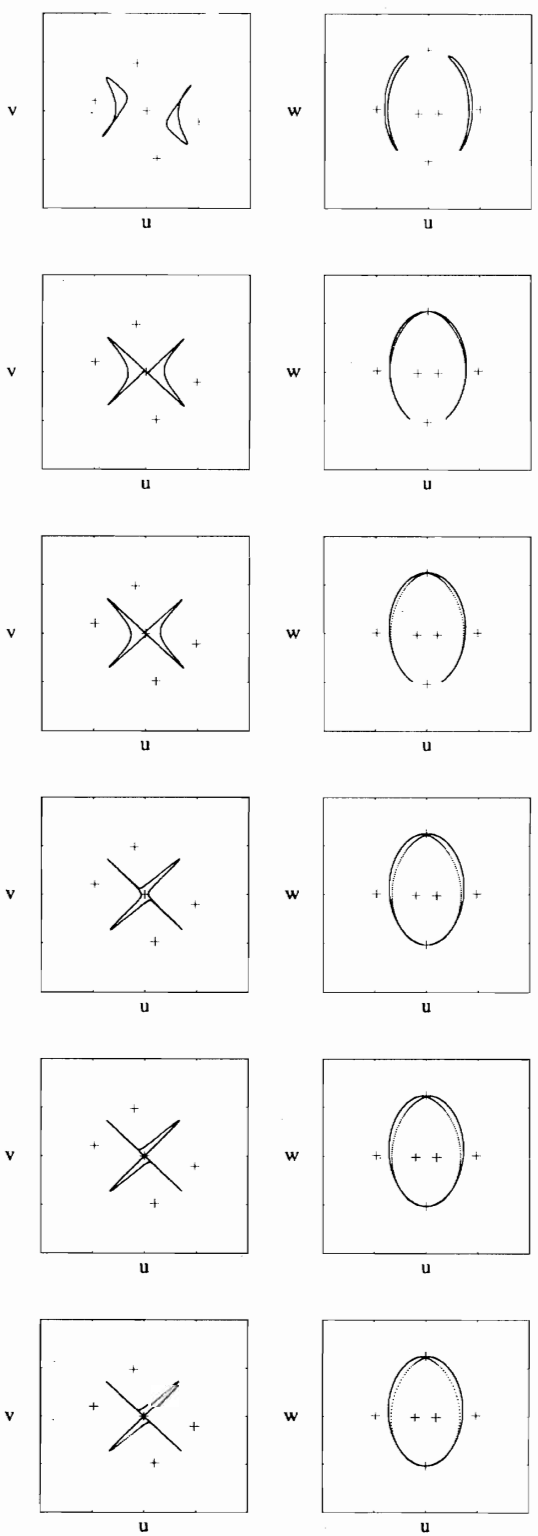

FIG. 9. Modulated waves for the broken- $D_{4}$-symmetry case, with $\mu=0.05, \Delta \mu=0.005, \Delta \omega=0.0003, R_{v}=-2$, $R_{w}=-1.75, \alpha=0$, as $R_{u}$ is varied from -1.96 to -2.0233 . Note the $\kappa_{1}$-symmetric modulated wave that forms near $R_{u}=$ -2.007 (frame 3 ). A homoclinic connection occurs between $R_{u}=-2.0231$ and -2.0233 (frames 5 and 6 ). waves involving the traveling wave fixed points. In the $D_{4}$-symmetric case we find a stable pair of periodic orbits that break the $\kappa_{1}$ and $\kappa_{2}$ symmetry, but that are invariant under $\kappa_{1} \kappa_{2}$. As the parameter $R_{v}$ is varied, these orbits eventually merge in a heteroclinic connection involving the pair of traveling wave fixed points at $( \pm u, 0,0)$. A new pair of $\kappa_{1}$-symmetric orbits then emerges. The global bifurcation thus leads to a $\kappa_{1} \kappa_{2} \rightarrow \kappa_{1}$ symmetry exhange. See Fig. 10. This picture is significantly modified when the $D_{4}$ symmetry of the problem is weakly broken $(\Delta \mu=0.001, \Delta \omega=0.005)$. For the parameter values examined there exists, in addition to an initial pair of orbits (which are still related by $\kappa_{1}$, but which are no longer $\kappa_{1} \kappa_{2}$-symmetric), a stable $\kappa_{1}$-symmetric orbit. Each of the orbits in the pair becomes homoclinic to a (different) traveling wave fixed point and is destroyed. The stable $\kappa_{1}$-symmetric orbit remains. See Fig. 11.

We note also that, as for Eqs. (5a,b) [17,18], stable modulated waves can coexist with stable traveling waves. This is true despite the fact that both such solutions can be brought arbitrarily close to the origin (as can be seen by rescaling the $u, v, w$ variables along with the unfolding parameters $\mu, \Delta \mu, \Delta \omega)$. We illustrate this in Fig. 12 .
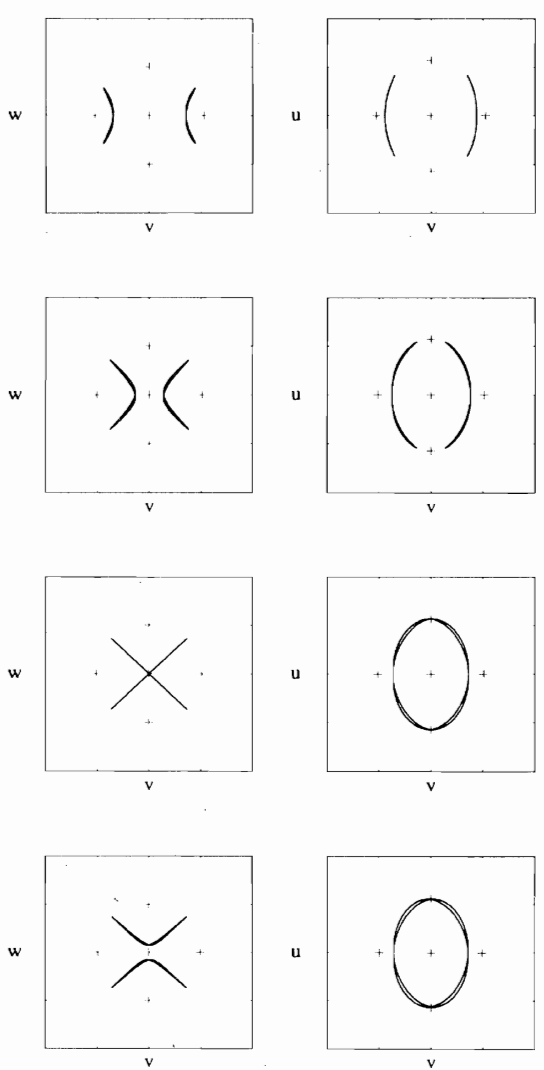

FIG. 10. Modulated wave for the $D_{4}$-symmetric case, with $\mu=0.05, \Delta \mu=0, \Delta \omega=0, R_{u}=-1.75, R_{w}=-2, \alpha=1.5$. The sequence shows the projections onto the $(v, w)$ and $(v, u)$ planes as the parameter $R_{v}$ is varied from -1.90 to -2.02 . Observe the heteroclinic connection in frame $3\left(R_{v}=-2.0\right)$. 

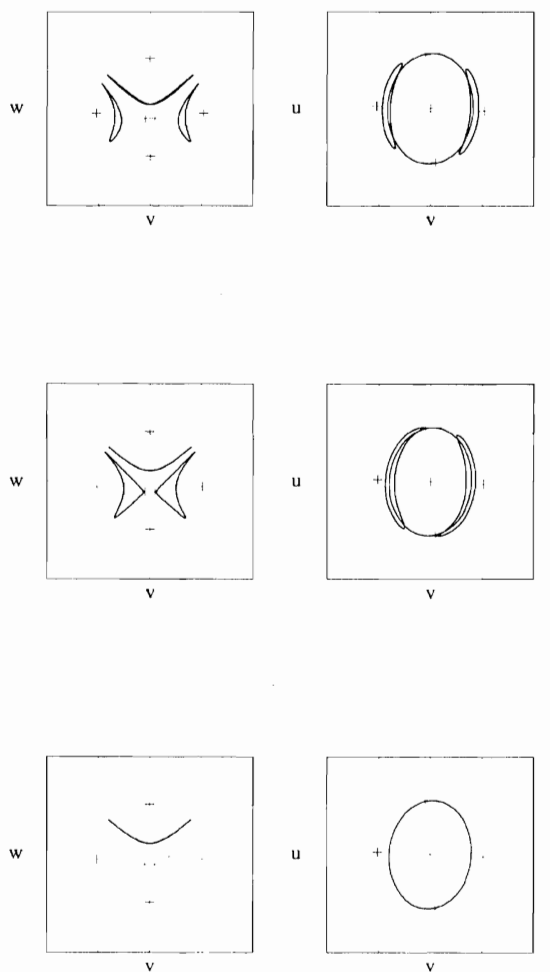

FIG. 11. Modulated waves for the broken- $D_{4}$-symmetry case, with $\mu=0.05, \Delta \mu=0.001, \Delta \omega=0.005, R_{u}=-1.75$, $R_{w}=-2, \alpha=1.5$, as the parameter $R_{v}$ is varied from -1.900 to -1.939

Numerical simulations also show that the stable modulated waves appearing in the $D_{4}$-symmetric problem persist even after the $D_{4}$ symmetry is (weakly) broken; the waves merely deform, despite the apparent absence of an invariant sphere.

\section{Period doublings, chaos, and collapsing states}

Numerical simulations have revealed a number of other dynamical phenomena associated with the modulated
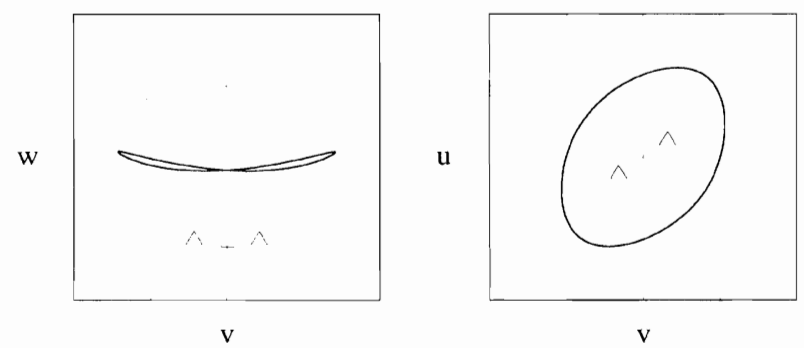

FIG. 12. A stable, $\kappa_{1}$-symmetric modulated wave coexisting with a stable traveling wave (denoted by a triangle), for $\mu=0.09, \Delta \mu=-0.01, \Delta \omega=0.1138, R_{u}=-1, R_{v}=-3$, $R_{w}=-2, \alpha=1.3$.
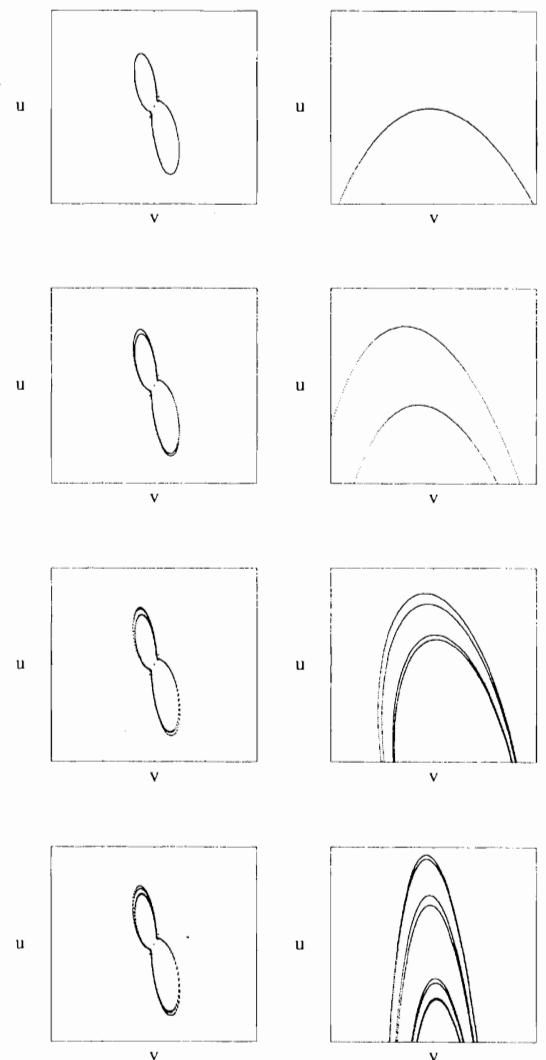

FIG. 13. Period doubling of a modulated wave for $\mu=0.1$, $\Delta \mu=-0.13, \Delta \omega=0.08, R_{v}=-0.9, R_{w}=-1.8, \alpha=2.5$ [Eqs. $(39 \mathrm{a}-\mathrm{c})]$, as the parameter $R_{u}$ is varied from 0.0610 to 0.0621 . The motion is projected onto the $(v, u)$ plane (left column); blow-ups of the top portion of each figure are provided (right column).

wave solutions. In particular, period-doubling sequences can occur for the modulated wave solutions. Since the modulated waves are in fact 2 -tori in the full fourdimensional phase space, such bifurcations correspond to torus doubling. Nonetheless, for visualization purposes, it is simpler to remain within the three-dimensional phase

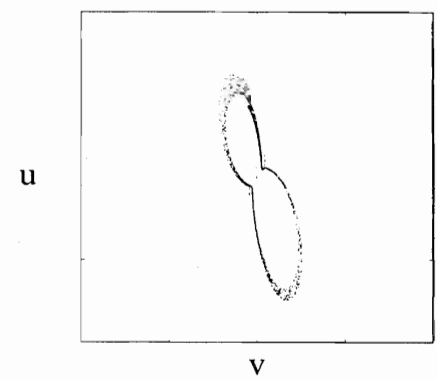

FIG. 14. A chaotic attractor for $R_{u}=0.0625$. The attractor breaks the reflection symmetry $\kappa_{1}$ of the problem (not obvious from picture). 
space (e.g., $u, v, w)$. In Fig. 13 we illustrate a sequence of period doublings, through period eight, as a control parameter $\left(R_{u}\right)$ is varied. Note that the modulated waves depicted here break the reflection symmetry $\kappa_{1}$ of the problem.

As the parameter $R_{u}$ is increased further such perioddoubling sequences can lead to (apparently) chaotic dynamics. This is depicted in Fig. 14. With increasing parameter values such an asymmetric attractor can grow and merge with its opposite parity counterpart to form a $\kappa_{1}$-symmetric object. This process can reverse as well. The existence of chaotic structures has also been noted by Knobloch and Hirschberg in a numerical study of the related equations $(5 \mathrm{a}, \mathrm{b})[19]$.

For other parameter values additional types of solutions are found. Figures $15(\mathrm{a}, \mathrm{b})$ depict large-amplitude periodic and apparently chaotic solutions. The presence of such large-amplitude solutions is particularly interesting, since points on these trajectories spend a great deal of time near the origin, while making occasional, shortlived, large-amplitude excursions away from it. During such "bursts," the amplitudes of the solutions have been observed to grow to over 40 times their characteristic size near the origin before undergoing a rapid collapse. This bursting behavior is qualitatively similar to the "repeated transients" and "collapse states" observed in experiments and numerical simulations of binary fluid convection $[7$, 8], and offers a possible explanation for the observed behavior. It is useful to examine these large-amplitude orbits in terms of the full four-dimensional normal form equations $(24 \mathrm{a}, \mathrm{b})$. In Figs. $16(\mathrm{a}, \mathrm{b}, \mathrm{c})\left|z_{1}\right|^{2}+\left|z_{2}\right|^{2}$ is plotted as a function of time for both types of solutions. The bursting behavior is clearly seen. Observe that the collapse of the spike is more rapid than its growth, as observed in the experiments $[7,8]$. Note that this representation for the solutions eliminates the fast oscillations at frequency near $\omega$, and hence describes the dynamics of their envelopes. Consequently, the temperature field $\vartheta(x, y, t)$ corresponding to Fig. $16(\mathrm{a})$ is in fact quasiperiodic. As noted previously, parameter values can be found such that the burst peaks are significantly larger than those depicted here. We have observed that the amplitudes of the bursts are highly sensitive to parameter value and integration time step, suggesting that in the physical system, external noise could play a prominent role. This behavior is reminiscent of that usually associated with the presence of a global bifurcation.

It should be noted that the "large-amplitude" bursting behavior considered here must really be regarded as small in absolute magnitude, in order for the normal form equations to remain valid; the significant feature of the solution is the large ratio of the amplitude of the bursts to the amplitude of the motion near the origin. The overall amplitude can be made small by reducing the bifurcation parameter and rescaling the equations. However, experimentally it is unclear whether the observed finite-amplitude bursting behavior would be reduced in amplitude if the system parameters were brought closer to threshold. If this is so the asymptotic description provided by the normal form equations presents a reasonable explanation for this particular phenomenon. However, if
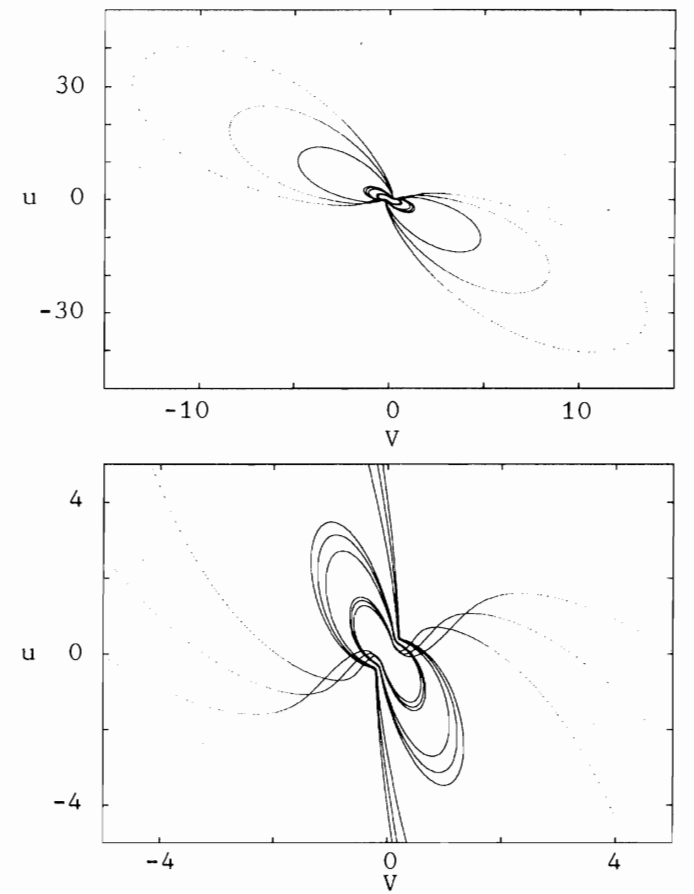

(a)
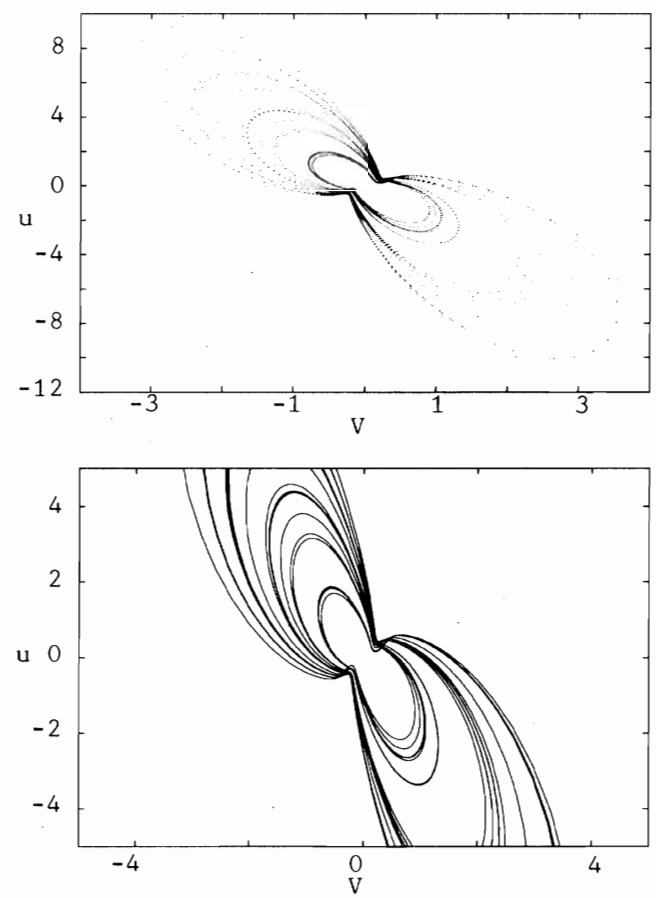

(b)

FIG. 15. Large-amplitude attracting orbits for $\mu=0.1$, $\Delta \omega=0.08, R_{u}=0.1, R_{v}=-0.9, R_{w}=-1.8, \alpha=2.5 ;(\mathrm{a})$ periodic attractor at $\Delta \mu=-0.13$; (b) aperiodic attractor at $\Delta \mu=-0.14$. Enlargements near the origin are also shown. In these parameter regimes the system behavior depends sensitively on the time step size used in the numerical integration scheme. The attractors shown here were calculated with a fourth-order Runge-Kutta method with a step size of $10^{-5}$. 


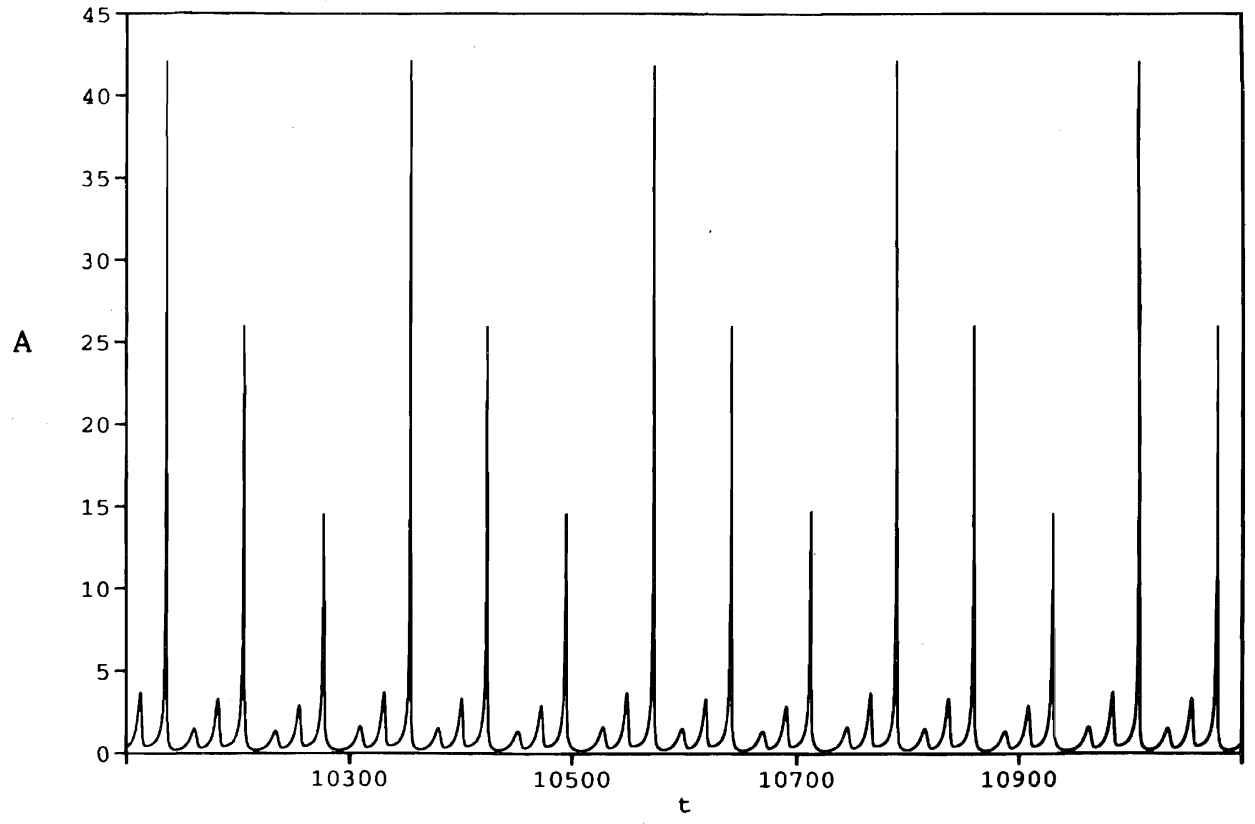

(a)

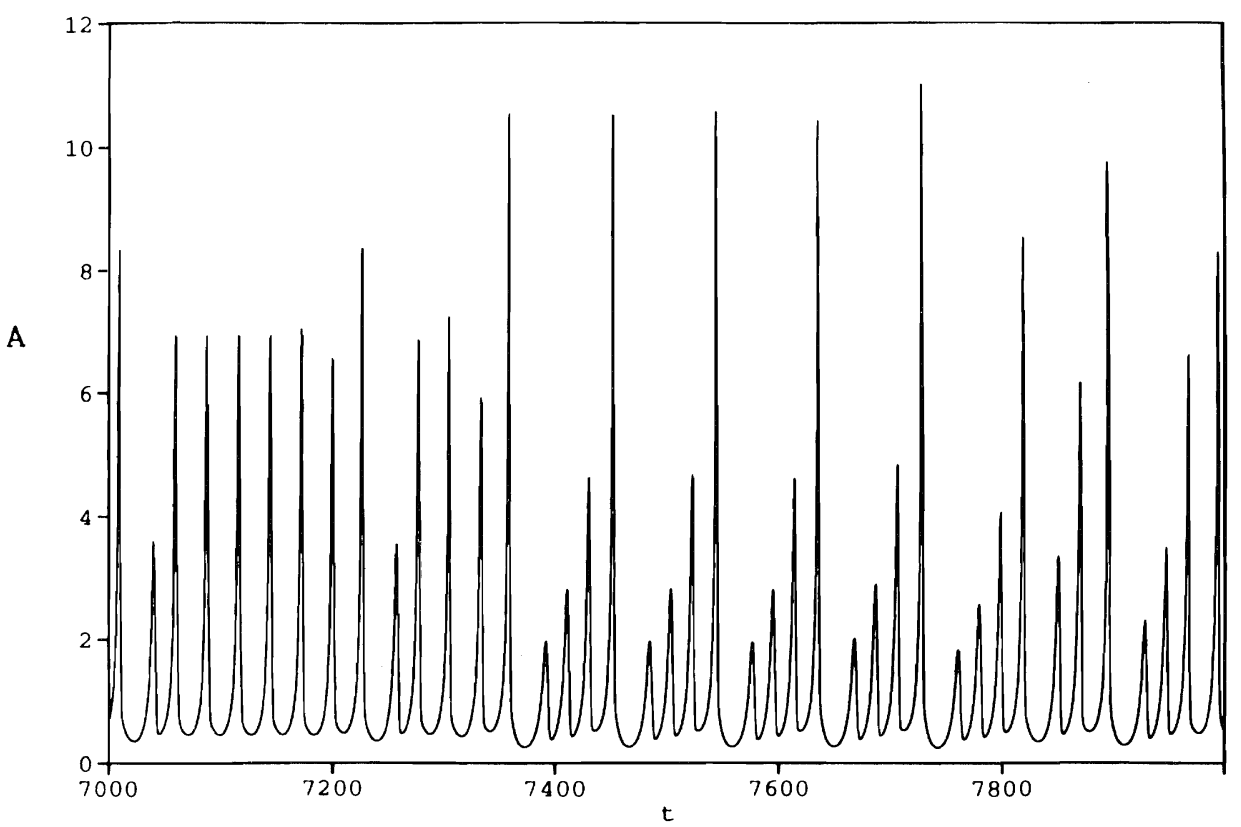

FIG. 16. The amplitude $A\left(=\left|z_{1}\right|^{2}+\left|z_{2}\right|^{2}\right)$ vs $t$ for the (a) periodic and (b) aperiodic "collapse" states depicted in Fig. 15. A close-up of the bursting behavior [for case $(a)]$ is shown in (c).

(b)

the experimental bursting behavior is inherently a finiteamplitude effect, then the theory offers no such solution.

\section{DISCUSSION AND CONCLUSIONS}

In this paper we have argued that traveling wave convection in a large aspect ratio container cannot be completely described as a perturbation of the unbounded translation-invariant system. In particular we have shown that when the problem is described as the large aspect ratio limit of oscillatory convection in a box the resulting amplitude equations have an approximate $D_{4}$ symmetry. As a consequence an additional cubic nonlinearity with an $O(1)$ coefficient is present. This new term is intrinsic to the problem and thus does not represent a perturbation to the unbounded problem, where this term is entirely absent. Mathematically this is because in finite boxes translation invariance cannot be used to argue for the absence of terms of the form $\left(\bar{v} \bar{w}^{2}, \bar{w} \bar{v}^{2}\right)$ in $(5 \mathrm{a}, \mathrm{b})$. A similar observation applies to the onset of steady con- 


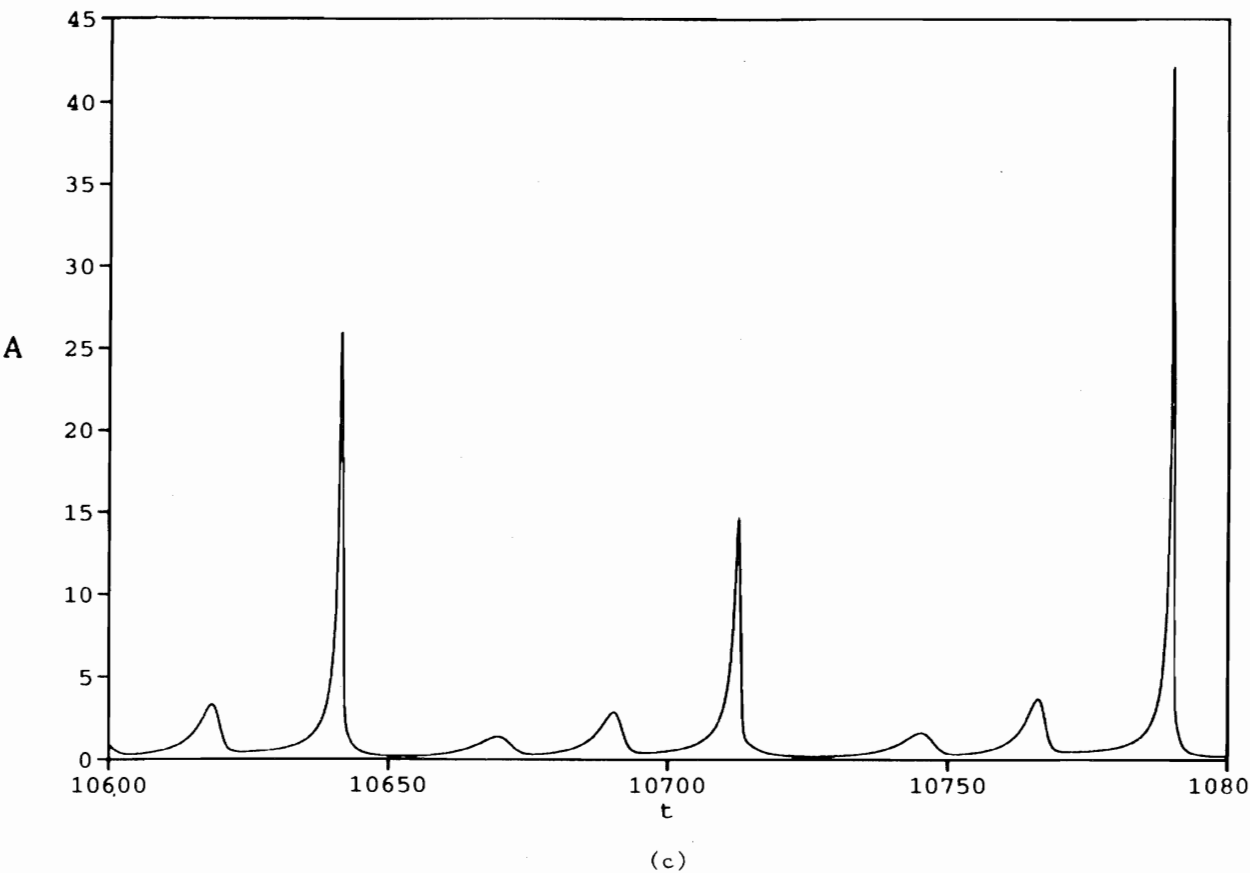

FIG. 16. (Continued).

vection in a large container. Here too the interaction between even and odd parity modes is described by amplitude equations with approximate $D_{4}$ symmetry [31] and the equations of [23] cannot be obtained as a small perturbation of the translation-invariant system.

The above conclusion is at variance with earlier attempts at treating this problem in terms of traveling waves using coupled complex Ginzburg-Landau equations with appropriate (linearized) boundary conditions for the envelope functions $[15,16]$. As shown in Ref. [16] this approach leads, near onset, to the normal form equations for the unbounded problem, perturbed by small linear terms. The predictions of the resulting theory $[17,18]$ are in good qualitative agreement with the experiments $[3,16]$ and with numerical integration of the GinzburgLandau equations [15]. Nonetheless, in spite of this success, there has in fact been no quantitative tests of this theory, neither against experiments nor against numerical simulations of the full fluid equations in long but finite boxes.

The present theory, as shown in this paper, also predicts states that bear a qualitative resemblence to the observed ones. In particular we have described not only the two types of standing waves and secondary bifurcations to traveling and blinking states, but have also found in our equations asymmetric quasiperiodic states of the type described by Cross [15], as well as states resembling the "repeated transients" first observed in Ref. [7].

Although once again the agreement is merely qualitative, the amplitude equations derived here have succeeded in describing a larger variety of the observed dynamical behavior. Despite this success, there remain some problems in directly comparing these results with the experimental observations. A prime difficulty is that in experiments on doubly diffusive and binary fluid con- vection, the precise degree to which the system is above threshold (corresponding to the parameters $\mu, \Delta \mu$ ), as well as the scaling behavior of the system, are not easily determined. Moreover, we know that for most values of the separation ratio the bifurcation to standing waves is in fact subcritical $[32,33]$ and this is so also in finite but large aspect ratio boxes [8]. However, for other problems, like the Taylor-Couette, the transition to standing waves is usually supercritical [34] and the potential for comparing the present theory with experiments is much improved. It should be emphasized that because of the approximate $D_{4}$ symmetry the applicability of our equations will extend beyond the present set of problems. We mention just two examples, the dynamics of Faraday waves in a nearly square container [35] and the long-term dynamics of the solar sunspot cycle arising from the interaction of dipole and quadrupole magnetic fields [36].

In an accompanying paper [37] we compute explicitly the normal form coefficients $A, B, C$ in Eqs. (24a,b) for a doubly diffusive system in Hele-Shaw geometry, recently studied in Ref. [4]. These calculations verify that all three coefficients remain of order one in the limit of large aspect ratio boxes and provide an independent justification of our approach. A related calculation by Zangeneh [38] for oscillatory magnetoconvection in finite containers yields similar conclusions.

\section{ACKNOWLEDGMENTS}

This work was supported in part by INCOR funds from Los Alamos National Laboratory and the National Science Foundation under Grant No. DMS-9406144. We are grateful to P. Hirschberg for numerous discussions and $\mathrm{H}$. Riecke and W. Nagata for bringing Refs. [20] and [38] to our attention. 
[1] R.W. Walden, P. Kolodner, A. Passner, and C.M. Surko, Phys. Rev. Lett. 55, 496 (1985).

[2] J. Fineberg, E. Moses, and V. Steinberg, Phys. Rev. Lett. 61, 838 (1988); P. Kolodner and C.M. Surko, ibid. 61, 842 (1988).

[3] P. Kolodner, C.M. Surko, and H. Williams, Physica D 37, 319 (1989); V. Steinberg, J. Fineberg, E. Moses, and I. Rehberg, ibid. 37, 359 (1989).

[4] A.A. Predtechensky, W.D. McCormick, J.B. Swift, Z. Noszticius, and H.L. Swinney, Phys. Rev. Lett. 72, 218 (1994); A.A. Predtechensky, W.D. McCormick, J.B. Swift, A. Rossberg, and H.L. Swinney, Phys. Fluids 6, 3923 (1994).

[5] C.D. Andereck, S.S. Liu, and H.L. Swinney, J. Fluid Mech. 164, 155 (1986).

[6] A.E. Deane, E. Knobloch, and J. Toomre, Phys. Rev. A 37, 1817 (1988).

[7] T.S. Sullivan and G. Ahlers, Phys. Rev. A 38, 3143 (1988): P. Kolodner, Phys. Rev. E 47, 1038 (1993).

[8] D. Jacqmin and J. Heminger (unpublished).

[9] E. Knobloch, Phys. Rev. A 34, 1538 (1986).

[10] Throughout this paper we describe symmetry properties of the various solutions in terms of the temperature eigenfunctions (as if for the Rayleigh-Bénard problem) instead of the stream function. This is because the former is a proper scalar while the latter is a pseudoscalar with respect to reflection.

[11] P. Coullet, S. Fauve, and E. Tirapegui, J. Phys. Lett. (Paris) 46, L787 (1985).

[12] E. Knobloch and J. De Luca, Nonlinearity 3, 975 (1990).

[13] M.C. Cross, Phys. Rev. Lett. 57, 2935 (1986).

[14] C.S. Bretherton and E.A. Spiegel, Phys. Lett. A 96, 152 (1983).

[15] M.C. Cross, Phys. Rev. A 38, 3593 (1988); M.C. Cross and E.Y. Kuo, Physica D 59, 90 (1992).

[16] G. Dangelmayr, E. Knobloch, and M. Wegelin, Europhys. Lett. 16, 723 (1991).

[17] G. Dangelmayr and E. Knobloch, in The Physics of Structure Formation: Theory and Simulation, edited by W. Güttinger and G. Dangelmayr (Springer-Verlag, Berlin, 1987), pp. 387-393.
[18] G. Dangelmayr and E. Knobloch, Nonlinearity 4, 399 (1991).

[19] E. Knobloch (unpublished); P. Hirschberg and E. Knobloch, Physica D, 90, 56 (1996).

[20] M. Bestehorn, R. Friedrich, and H. Haken, Z. Phys. B 77, 151 (1989).

[21] P. Hirschberg and E. Knobloch (unpublished).

[22] M.G.M. Gomes and I. Stewart, in Dynamics, Bifurcation and Symmetry: New Trends and New Tools, edited by P. Chossat (Kluwer, Norwell, MA, 1994).

[23] E. Knobloch and J. Guckenheimer, Phys. Rev. A 27, 408 (1983).

[24] D. Armbruster, J. Guckenheimer, and P. Holmes, SIAM J. Appl. Math. 49, 676 (1987).

[25] L.S. Tuckerman and D. Barkley, Physica D 46, 57 (1990).

[26] C. Elphick, E. Tirapegui, M.E. Brachet, P. Coullet, and G. Iooss, Physica D 29, 95 (1987).

[27] J.W. Swift, Nonlinearity 1, 333 (1988).

[28] W. Nagata, Proc. R. Soc. Edinburgh A 117, 1 (1991).

[29] Note that these equations correspond to a degenerate case of the nonresonant double Hopf normal form equations. See J. Guckenheimer and P. Holmes, Nonlinear Oscillations, Dynamical Systems, and Bifurcations of Vector Fields (Springer-Verlag, New York, 1986), for a description of the generic case.

[30] T. Clune and E. Knobloch, Physica D 74, 151 (1994).

[31] P. C. Hirschberg, Ph.D thesis, University of California at Berkeley (1994).

[32] W. Schöpf and W. Zimmermann, Europhys. Lett. 8, 41 (1988).

[33] T. Clune and E. Knobloch, Physica D 61, 106 (1992).

[34] Y. Demay and G. Iooss, J. Mécanique Th. Appl., Numéro spécial, 193 (1984).

[35] F. Simonelli and J.P. Gollub, J. Fluid Mech. 199, 471 (1989).

[36] A.S. Landsberg and E. Knobloch, Mon. Not. R. Astron. Soc. 278, 294 (1996).

[37] A.S. Landsberg and E. Knobloch, following paper, Phys. Rev. E 53, 3601 (1996).

[38] H.R.Z. Zangeneh, Ph.D thesis, University of British Columbia (1993). 